\title{
11 Textstrategien und Argumentationsmuster
}

\subsection{Kommunikative Strategien und argumentative Elaboration}

Im Gegensatz zu rechtsradikalen oder fundamentalistischen Schreibern, die in ihren Äußerungen judenfeindliche Polemik vor allem mittels Beschimpfungen und manifester Diskriminierungsformen ausdrücken, geben sich die gebildeten Verfasser (von denen die meisten einen Hochschulabschluss und zum Teil akademische Titel haben) viel Mühe, ihre antisemitischen Einstellungen mittels argumentativer Strategien zu verteidigen und zu begründen. Eine bloße Beschimpfung durch das Wort Saujude oder eine Drohung wie Ihr werdet alle krepieren! lässt keine Argumentation erkennen. Die Texte ${ }^{1}$ der akademischen Verfasser jedoch sind lang, weisen zahlreiche Argumentationsmuster auf und sind sprachlich-stilistisch oft auf einem hohen Niveau. In den textuellen Elaboraten aber werden, wie bereits in Kap. 5 erörtert, oft dieselben tradierten Stereotype kodiert, wie sie bei Extremisten zu finden sind. Dass die eigene, verbal ausgedrückte und in der Analyse klar erkennbare antisemitische Haltung als solche dabei fast immer vehement geleugnet wird, ist typisch für diese Textproduzenten. Die Leugnung selbst ist hier als eine Strategie zu betrachten, die der Selbstlegitimation (und damit der Rechtfertigung für die Artikulation der ressentimentbelasteten Äußerungen) dient (s. Kap.11.2 und 11.4).

Um den Zusammenhang von mentaler Einstellung, kommunikativer Funktion und sprachlicher Realisierung bei diesem Typ des Verbal-Antisemitismus klar machen zu können, bedarf es einer kurzen Erörterung der linguistischen Grundbegriffe „Funktion“, „Strategie“ und „Mittel“. Die kommunikative Funktion einer sprachlichen Äußerung entspricht ihrem Handlungswert. Mit einer Äußerung wie Juden haben zu viel Einfluss im Finanzsektor wird eine allgemeine Aussage getroffen, mit der der Schreiber eine bestimmte Funktion (z. B. Kritik oder Entwertung) realisieren will. Die kommunikative Funktion einer Äußerung geht über die Bedeutung der Äußerung hinaus und ist die Handlung, die vom Produzenten mit der Äußerung vollzogen wird (vgl. Schwarz/Chur 52007: 28-32).

\footnotetext{
1 Um einem möglichen Missverständnis vorzubeugen: Analysiert werden in diesem Kapitel ausschließlich Texte, die als klar verbal-antisemitisch eingestuft worden sind, also judenfeindliche Stereotype und Argumente enthalten. Hiervon abzugrenzen sind Schreiben, die tatsächlich ernst gemeinte Sorge und legitime Kritik ausdrücken, ohne anti-jüdische Inhalte zu verbalisieren. Zum Teil ähneln sich diese Texte in ihrer sprachlichen Gestaltung. Erst eine kognitions- und textlinguistische Analyse macht den Unterschied zwischen VerbalAntisemitismus und z. B. Israel-Kritik deutlich (s. hierzu Kap. 7).
} 
Dabei ist zwischen der intendierten (beabsichtigten) Funktion des Sprachproduzenten und den potenziellen Funktionen, die die Äußerung zusätzlich (für die Rezipienten) haben kann, zu unterscheiden.

So haben NS-Vergleiche (z. B. Scharon ist der Hitler des Nahen Ostens, Israel ist ein NS-Staat) die Funktion, den/das Angegriffene(n) besonders drastisch zu kritisieren bzw. dem verglichenen Referenzbereich besondere Bedeutung zuzuschreiben (z. B. Pogromstimmung in Palästina, Palästinenser werden heute so schikaniert wie damals die Juden). Mit jedem NS-Vergleich aber wird auch automatisch über die involvierte und unverhältnismäßige Analogie nicht nur die NS-Zeit relativiert, sondern es werden auch ihre Opfer verhöhnt. Der NS-Zeit wird dadurch, intentional oder nicht-intentional, der Status des Singulären, des Präzedenzlosen genommen.

Die Realisierung kommunikativer Funktionen erfolgt mittels bestimmter verbaler Strategien. ${ }^{2}$ Verbale Strategien sind kommunikative Handlungsmuster. Eine Strategie S (z. B. die der Täter-Opfer-Umkehr) wird eingesetzt, um die Funktion F (z. B. Kritik am Staat Israel und darüber hinaus dessen Delegitimierung) zu realisieren und eine Reaktion R (z. B. die emotionale Empörung oder handlungsorientiert die Akzeptanz des Boykotts israelischer Waren) beim Rezipienten $\mathrm{zu}$ erreichen. Strategisch $\mathrm{zu}$ handeln heißt, in Hinblick auf eine bestimmte (kommunikative) Funktion bestimmte Argumente und sprachliche Mittel nach deren Eignung auszuwählen. So kann die Funktion Kritik (je nach strategischem Kalkül) direkt als Beschimpfung oder indirekt als Ironie oder als rhetorische Frage realisiert werden, um den Rezipienten zu beleidigen, anzugreifen oder $\mathrm{zu}$ überzeugen. Damit stellen Strategien Ziel-Mittel-Relationen dar und lassen sich nach generalisierten Funktionen (Kritisieren, Disqualifizieren, Schuld abwehren, Leugnen usw.) mit Zielsetzungen (Beleidigen, Diskriminieren) benennen. Während rechtsradikale Schreiber auf Beleidigungen und Diskriminierungen abzielen und diese entsprechend radikal mittels direkter Sprechakte (Beschimpfungen, Verhöhnungen, Drohungen) verbalisieren (s. hierzu Kap. 10.1), möchten die Antisemiten der Mitte erstens ihr Gesicht wahren und zweitens die Rezipienten davon überzeugen, dass sie mit ihrer Kritik Recht haben.

2 Vgl. hierzu auch Brinker (1986: 180), Hoffmann (1998: 63), Ortak (2004: 135), SchwarzFriesel (2007: 198). Sowohl der Funktions- als auch der Strategiebegriff sind nicht nur auf eine einzelne Äußerung zu beziehen, sondern auch auf größere Textteile oder einen gesamten Text. So lassen sich lokale und globale Strategien danach unterscheiden (vgl. auch Jucker 1986: 186), ob sie in einem einzelnen Sprechakt oder über eine größere Anzahl von sprachlichen Mustern realisiert sind. Ebenso kann zwischen der kommunikativen Funktion (Illokution) einer einzelnen Äußerung und der kommunikativen Funktion eines Textes (Globalillokution) differenziert werden. 
Die Strategie der Täter-Opfer-Umkehr (die Juden nicht als Opfer, sondern als Täter darstellt) z. B. basiert aktuell auf der Konzeptualisierung, dass die Juden heute durch ihr schamloses, den deutschen Staat ausnutzendes Verhalten Täter und die Deutschen als Bürger dieses Staates Opfer sind bzw. dass die Israelis trotz ihrer jüdischen Herkunft und der Vergangenheit ihrer Eltern etc. nun zu Tätern an den Palästinensern, den neuen Opfern, geworden sind. Die Funktion dieser Täter-Opfer-Umkehr-Strategien liegt zum einen in der Kritik bzw. Diffamierung von Juden und/oder Israelis, zum anderen in der Relativierung der deutschen Schuld (auch wenn diese Relativierung nicht immer intendiert wird).

Inhaltlich basieren Strategien somit auf geistigen Repräsentationen. Bei der Täter-Opfer-Umkehr-Strategie sind dies entweder die Glaubensinhalte 'Israelis benutzten exzessive Gewalt. Sie haben nichts aus der Vergangenheit gelernt. Sie haben keine moralische Größe erworben.' und/oder 'Juden sind nicht wirklich Bürger des deutschen Staates. Ihre Solidarität gilt Israel. Sie nutzen den deutschen Staat aus.'

Solche Informationsstrukturen finden sich im antisemitischen Diskurs auch einzeln als Argumente ${ }^{3}$ verbalisiert. Argumente sind in der Regel sprachlich kodierte „Beweisführungen“, die meist in Form von mehreren Aussagen realisiert werden. Häufig repräsentieren sie pseudo-kausale Zusammenhänge, z. B. in der Art: 'Juden sind von Natur aus böse und habgierig. Die Israelis sind Juden, daher böse und habgierig, und sie haben den Palästinensern das Land geraubt. Deutschland ist für die Not der Palästinenser mitverantwortlich, da es den Staat Israel unterstützt (hat). Als Deutsche(r) hat man nun besondere Verantwortung und muss sich also für die armen Palästinenser einsetzen.'

Argumente sind also aus der Sicht des Sprachproduzenten wahre und begründete Behauptungen/Feststellungen, d.h. sie setzen ihre Rechtfertigungsfähigkeit voraus. Dabei können drei Typen von Argumenten ${ }^{4}$ unterschieden werden:

3 In der Rhetorik wird unter einer Argumentation das Finden passender Argumente und deren Verbindung zu einer überzeugenden Argumentationskette verstanden (vgl. Ottmers ${ }^{2} 2007$ : 66). Den Ausgangspunkt einer Argumentation bildet ein strittiger (oder als strittig erachteter) Sachverhalt, der in seinem Geltungsanspruch unstrittig gemacht werden soll. Hierfür werden als unstrittig erachtete Argumente (Informationen, Behauptungen) angeführt, die den Sachverhalt entweder stützen oder ihn widerlegen (vgl. Ottmers ${ }^{2} 2007: 68-70$, 72). Im sprachwissenschaftlichen Sinn kann mit Herbig (1992: 62) unter Argumentieren eine Sprachhandlung verstanden werden, „mit de[r]en Hilfe ein Sprecher aufgrund seiner Situationseinschätzung den für einen oder mehrere Adressaten erkennbaren Versuch unternimmt, diese(n) mit Hilfe einer oder mehrerer unterstützenden Äußerungen zu bewegen, tatsächliche oder nur der Situationseinschätzung nach ‘strittige' Geltungsansprüche der Wahrheit oder Richtigkeit von Äußerungen zu akzeptieren.“

4 Vgl. zur Argumentationsanalyse auch Toulmin (1958), Kopperschmidt (1989), Herbig (1992) und Bayer (2007). 
Faktische Argumente sind verifizierbar (durch historisches Wissen, Dokumente, Resolutionen etc.) und auf ihren Wahrheitswert hin prüfbar (z. B. Gegen Israel wurden $x$ Resolutionen bei der UN eingereicht.), konzeptuelle Argumente (Plausibilitätsargumente) dagegen basieren allein auf Glaubensinhalten bzw. auf als allgemeingültig unterstellten Annahmen (z. B. Der gesunde Menschenverstand sagt doch wohl, dass ... oder Niemand wird diesen/einen unmoralischen Staat unterstützen wollen ...). Scheinargumente/Pseudoargumente werden aus strategischen Gründen (wider besseres Wissen) benutzt (z. B. werden Meinungen als Tatsachen ausgegeben wie in Israel, da sind sich alle einig, ist ein Apartheidstaat oder die eigene Person wird als Autorität aufgeführt wie in Ich bin fünfmal in Israel gewesen, Sie können mir daher getrost glauben ...).

Die Strategie des Zitierens von Autoritäten (auch XY spricht vom Vernichtungskrieg) beispielsweise dient wie in (1) und (2) der Legitimierung der eigenen Position. Diese Absicherung ist die kommunikative Funktion der Strategie, die auf dem faktischen Argument 'Auch Prominente/Experten sehen das so' basiert.

„Moshe Zimmermann sprach am 14.Mai in Tübingen (Lucas-Preis) von einem 'nazistischen Potenzial eines bestimmten Gesellschaftssektors in Israel'.“[ZJD_05.06.2002_Rud_001]

„Der Politikwissenschaftler und Professor an der Bar Ilan Universität Tel Aviv, Menachem Klein, äusserte kürzlich in einem Interview öffentlich: [...]“[ZJD_22.04.2002_Gie_001]

Verbal-antisemitische Strategien basieren in der Regel auf mehreren Argumenten, um ihre Wirksamkeit zu erhöhen. Die Strategie der Delegitimierung Israels wird z. B. oft durch das faktische Argument 'UN-Resolutionen', das konzeptuelle Argument 'Massenvernichtungswaffen' sowie die Scheinargumente 'Landraub', 'Staatsterrorismus' oder 'Apartheid' etc. gestützt.

Jede Strategie kann nun mit unterschiedlichen sprachlichen Mitteln (Lexeme, Syntax) realisiert werden. Die Strategien sind also nicht mit den eingesetzten sprachlichen Mitteln gleichzusetzen. So kann die Täter-Opfer-Umkehr-Strategie vulgärsprachlich und syntaktisch simplifiziert als „Jetzt seid ihr die Verbrecherschweine!“ [IBD_14.08.2006_Auf_001] zum Ausdruck kommen oder elaboriert und stilistisch gehoben als „Ihr Opferstatus ist nicht mehr aufrecht zu erhalten. Durch ihre brutale Gewaltanwendung [...] sind sie schuldig an Verbrechen gegen die Menschlichkeit“"ZJD_18.06.2002_Oeh_001]. 
Die Strategien des Verbal-Antisemitismus ${ }^{5}$ sind demzufolge charakterisierbar als kommunikative Handlungsmuster mit spezifischen Funktionen, die auf bestimmten Argumenten beruhen und je nach Produzent und/oder Kontext sprachlich unterschiedlich realisiert sein können.

Die Textanalysen des Korpus zeigen, dass Zuschriften von Verfassern aus der Mitte, die eine antisemitische Einstellung erkennen lassen, häufig ähnliche kommunikative Strategien, argumentative Muster ${ }^{6}$ und sprachliche Charakteristika aufweisen. Zum Teil entsteht aufgrund der frappierenden Ähnlichkeit der Textstrukturen der Eindruck, die Verfasser würden trotz aller individuellen Unterschiede hinsichtlich Alter, Geschlecht, Herkunft usw. wie miteinander verabredet auf abrufbare Schablonen zurückgreifen. Dies belegt aber nur, wie tief und wie homogen die kognitiven Stereotype und emotionalen Ressentiments gegen Juden aufgrund ihrer langen kulturellen Tradition in den Köpfen vieler Menschen kollektiv verankert sind.

Hinsichtlich ihrer kommunikativen Funktion lassen sich die sprachlichen

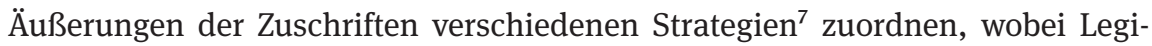
timations-, Vermeidungs-, Rechtfertigungs-, Relativierungs- sowie Aus- und Abgrenzungsstrategien $\mathrm{zu}$ unterscheiden sind. Zudem lassen sich charakteristische sprachliche Realisationsformen und argumentative Muster beschreiben. Eine klare Eins-zu-eins-Zuordnung von einem argumentativen Muster zu einer Strategie ist hingegen nicht immer möglich, denn je nach Kontext sind konkrete Verbalmanifestationen unterschiedlichen kommunikativen Funktionen zuzuordnen. So wird in Beispiel (3) die Angabe des Alters angeführt, um die persönliche Schuldlosigkeit an den deutschen Verbrechen im Nationalsozialismus zu belegen und somit auch gegenwärtig eine deutsche Verantwortlichkeit abzulehnen.

„Als im Jahr 1966 Geborener fühle ich mich nicht für die Verbrechen des 2. Weltkrieges verantwortlich [...].“ [ZJD_01.03.2002_Lue_001]

5 Hier handelt es sich um eine verkürzte Ausdrucksweise: Bei den Strategien des VerbalAntisemitismus geht es um die spezifischen Strategien der Sprachbenutzer, um Antisemitismus über bestimmte sprachliche (formale) Mittel zu äußern. Dagegen bezieht sich die Formulierung Antisemitismus als verbale Strategie auf die generelle Tendenz, dass eben die Sprache und nicht Karikaturen oder Grabschändungen benutzt werden, um antisemitische Ressentiments in der gesellschaftlichen Mitte auszudrücken. Antisemitismus als verbale Strategie zielt auf eine Entradikalisierung und Legitimierung durch das Ersetzen von nonverbalen Handlungen durch Verbalmanifestationen.

6 Wiederkehrende Argumente und Argumentfolgen können als argumentative Muster beschrieben werden.

7 Vgl. hierzu auch die Beschreibungskategorien von Wodak (1990), Wodak et al. (1990) und Reisigl/Wodak (2001) sowie van Dijk (2002). 
Hier steht die Funktion der Relativierung im Vordergrund. Eine Altersangabe kann aber auch erfolgen, um sich auf Erfahrungswissen zu berufen und verantwortungsvolles Handeln aufzuzeigen, wie in (4). Dabei dient die Information der Legitimierung des Schreibers.

„Ich bin 65. Mein Leben ist stets nach humanistischen Prinzipien ausgerichtet gewesen.“[ZJD_21.04.2005_Lan_001]

Da die jeweiligen Strategien in einem engen thematischen Zusammenhang stehen, sich zum Teil bedingen und ergänzen, kann es zu diversen Kopplungen und Überschneidungen kommen. Das Leugnen des eigenen Antisemitismus kann beispielsweise sowohl der Legitimation als auch der Vermeidung dienen. Dennoch lassen sich die Strategien innerhalb des Textaufbaus voneinander abgrenzen, und durch ihre Analyse wird die spezifische Argumentationsstruktur judenfeindlicher Texte transparent.

Im Folgenden werden die einzelnen Strategien anhand von Beispielen aus dem Korpus erläutert.

\subsection{Legitimierungsstrategien und offensive Selbsterhöhung: „Ich bin durch und durch Humanist!“}

Die globale Textstruktur einer Vielzahl der Zuschriften zeichnet sich im Einleitungsteil durch Legitimierungsstrategien aus, mit denen das Schreiben an sich gerechtfertigt und/oder einem möglichen Antisemitismus-Vorwurf vorgreifend entgegnet wird. ${ }^{8}$

Legitimierungsstrategien sind gekennzeichnet durch Argumente, die dem Aufbau eines positiven Selbstbildes dienen (positive Selbstdarstellung) ${ }^{9}$ und Respektabilität bezeugen sollen. Der Textproduzent stellt sich als demokratisch eingestellte, politisch korrekte sowie menschlich-moralisch integre Persönlichkeit dar und schreibt sich insbesondere Eigenschaften und Kompetenzen wie Erfahrungs- und Fachwissen, Rationalität und Objektivität zu. Eigenschaften, die

8 Die Textanalysen vollständiger Zuschriften zeigen, dass sich für die jeweiligen Textstrategien innerhalb der globalen Textstruktur ein Verteilungsmuster zu ergeben scheint: Zuerst Selbstlegitimation, dann Abgrenzungs-, Vermeidungs- und Relativierungsstrategien; in der Schlussphase dann vor allem Rechtfertigungsstrategien. Ein typischer Beispieltext, der dies exemplarisch veranschaulicht, befindet sich unter [ZJD_01.09.2006_Tef_001] im Anhang. 9 Bei Wodak et al. (1990: 353) wird die positive Selbstdarstellung als eine Strategie der Argumentation klassifiziert, mit der der Sprecher seine „Unschuld, Integrität, philosemitische Haltung etc. betont“. 
ihn seines Erachtens befähigen und berechtigen, sich mit seinem Anliegen an die Israelische Botschaft in Berlin und/oder an den Zentralrat der Juden in Deutschland zu wenden bzw. seine Gedanken und seine Meinung zu äußern und gleichzeitig als seriöser Absender und Gesprächspartner wahrgenommen zu werden.

Die positive Selbstdarstellung kann sprachlich wie folgt realisiert werden:

a) über Selbsteinschätzungen in Form von Zuordnungen positiver Attribute: Dies sind Aussagen zur eigenen Person, die mit Hilfe positiv wertender Selbstattribuierungen und Pronomen der 1. Person Singular sprachlich als Ich bin/tue $x$ realisiert werden (vgl. auch Wodak et al. 1990: 353). Wenn der Verfasser sich z. B. zu einem in demokratischen Gesellschaften positiv beurteilten Wert wie 'alle Menschen sind gleich' bekennt, sollen die Eigenschaften des Autors, tolerant und vorurteilsfrei zu sein, in den Vordergrund treten:

„Ich gehöre zu den Bürgern, bei der der Mensch ein Mensch ist und auch bleibt. Egal welcher Religion oder Weltanschauung er angehört.“ [ZJD_08.04.2002_Lud_001]

Quantitativ ist das Wort Humanist das am häufigsten benutzte Mittel zur Selbstbeschreibung: Das ist insofern signifikant, da diese selbstreferenzielle Einschätzung im Kontrast zu den Adressaten implizieren kann, dass Juden und/oder Israelis nicht humanistisch gesinnt seien (s. hierzu Kap. 5.2 und 10). Die Darstellung moralischer und/oder politischer Integrität erfolgt des Weiteren über die explizite Selbstverortung im sozialdemokratischen Einstellungsbereich und/oder den Verweis auf einen „höheren“ Wertekonnex wie Völkerverständigung, s. (6), und Weltfrieden, s. (7):

(6) „Sehr geehrte Damen und Herren, ich spreche hier in sozialdemokratischen Fundamenten und im Wunsch der friedlichen Völkerverständigung.“[IBD_25.06.2006_Rei_001]

„Ich bitte Sie im Namen des Friedens auf dieser Welt beenden Sie diesen sinnlosen Krieg.“ [ZJD_01.04.2002_Ask_001]

b) Eine spezifische Form der Selbsteinschätzung stellen biographische Auskünfte wie Altersangabe, Lebenslauf, Ausbildungsniveau bzw. Berufsbezeichnung sowie Angaben zum Bildungsstand und zur Informiertheit zum Thema „Juden“ und/ oder „Israel“ dar. ${ }^{10}$ Diese positive Selbstpräsentation erfolgt über den Verweis

10 Biographische Auskünfte treten aber nicht ausschließlich im Einleitungsteil (Legitimie- 
auf persönliches Erfahrungs- und Allgemeinwissen, auf gesellschaftspolitisches Interesse sowie auf spezielle Sachkenntnis.

So können beispielsweise Altersangaben einerseits auf die persönliche demokratische Gesinnung verweisen, wie in folgendem Beispiel (8), bei dem der Autor seine politische Gesinnung explizit macht, obwohl er referenziell distanzierend von sich in der 3. Person Singular spricht: ${ }^{11}$

(8) „Die Analyse, eines 27 jährigen deutschen, der politisch interessiert ist, und ein Aufrechter demokrat ist und für friedliches miteinander kämpft [...]“[IBD_18.02.2006_Bri_001]

Altersangaben können aber anderseits auch als Ausdruck von Lebenserfahrung benutzt werden, wobei sich die Schreiber dabei auf eine unterstellte Altersweisheit berufen, wie in (9):
„Ich will nicht behaupten, daß Herr Friedmann den von mir geschilder- ten Eindruck bewußt hervorruft, niemand kann ja bekanntlich aus seiner Haut, was ich als pensionierter Gymnasiallehrer sehr wohl weiß, da ich in dieser Hinsicht beruflich meine Erfahrungen gemacht habe (Jahrgang 1934).“[ZJD_06.06.2002_Hau_001]

Die explizite Altersangabe bei Schreibern der älteren Generation geht zudem oftmals mit einer emphatisch realisierten Abgrenzung vom Nationalsozialismus einher:

$$
\begin{aligned}
& \text { „Ich bin } 74 \text { Jahre alt und bei Gott kein Nazi.“ [ZJD_24.10.2006_Bon_001] } \\
& \text { „Ich bin jetzt 62 Jahre alt. Spätestens Mitte der 50er Jahre - als ich langsam } \\
& \text { die Deutsche Geschichte begriff - habe ich mich als Deutscher für unsere } \\
& \text { Vergangenheit geschämt.“ [IBD_22.07.2006_ano_003] }
\end{aligned}
$$

Explizite Angaben zum Bildungsweg, aber auch Berufsbezeichnungen werden genannt, um eine fundierte und nicht auf Vorurteilen beruhende Meinungsbildung, Einstellung oder Forderung zu belegen:

rungsstrategie) auf; s. hierzu auch Vermeidungsstrategien und deren Funktion. 11 Gleichzeitig dient bei jüngeren Generationen die Altersangabe auch der Entlastung von der historischen deutschen Schuld. 

„Mir sind die geschichtlichen Entwicklungen seit der Gründung Israels bekannt, und ich habe mich auch durch das Studium der Poli- tikwissenschaften immer wieder intensiv damit auseinandergesetzt.“ [IBD_10.06.2006_Sch_002]

Im nachfolgenden Beispiel (13) wird ferner Antisemitismus als eine mit geringem Bildungsgrad korrelierende Prädisposition dargestellt und somit als ein Bildungsproblem marginalisiert.
„als Mensch mit Bildung [...] und demzufolge auch nicht mit einer antise- mitischen 'Voreinstellung' versehen, möchte ich zu Ihren veröffentlich- ten Rechtfertigungsversuchen der oben genannten Art folgendes bemer- ken [...]“ [ZJD_08.04.2002_Ram_001]

Für den Textproduzenten ergibt sich ein quasi-syllogistischer Schluss ${ }^{12}$ mit der Oberprämisse 'Menschen mit Bildung sind keine Antisemiten'. Die Unterprämisse lautet 'ich bin ein Mensch mit Bildung', sodass als Konklusion abgeleitet wird 'weil ich ein Mensch mit Bildung bin und Menschen mit Bildung keine Antisemiten sind, bin ich kein Antisemit'. Während die Ober- und Unterprämisse implizit zu erschließen sind, markiert der Autor sprachlich explizit den Schlussfolgerungsprozess über die Kausalkonjunktion demzufolge.

Der Verweis auf eigenes Erfahrungswissen durch Begegnungen mit Juden sowie einer langwierigen Auseinandersetzung mit jüdischen und israelischen Themen wird ebenfalls als Beleg für eine vorurteilsfreie und sich auf die Realität beziehende Meinungsbildung herangezogen, wie in der E-Mail eines promovierten Akademikers aus Emden:
„seit Längerem beschäftige ich - Jahrgang 1930, in einem, dem einer jüdischen Familie benachbarten Einfamilienhaus aufgewachsen, mit deren einzigem, dem Holocaust durch Exil entkommenen Sohn ich mich vor drei Jahren traf - mit den Problemen des Judentums und Israels, aber auch mit den Problemen durch das Judentum und durch Israel.“ [IBD_21.01.2008_Seg_001]

12 Solche Rechtfertigungsmuster finden sich auch im öffentlichen Diskurs: Ein bekanntes Beispiel hierfür ist Norbert Blüms in diversen Talkshows artikulierte Aussage, er könne als Christ gar kein Antisemit sein (s. hierzu die Ausführungen in Kap. 4, die zeigen, wie absurd diese Aussage ist). 
c) Eine positive Selbstdarstellung erfolgt des Weiteren über Normenkongruenz, d. h. die allgemeingültige Bejahung von positiven, gesellschaftlich-politisch etablierten Normen. Im Unterschied $\mathrm{zu}$ a) und b) spricht der Textproduzent hierbei nicht in der 1. Person Singular von sich, sondern er identifiziert sich generalisierend über positiv wertende Aussagen mit einer Gruppe, sodass die individuelle Positivattribuierung implizit erfolgt. Im folgenden Beispiel wird der Wille zu einem friedvollen Zusammenleben der Religionen als allgemeines Ziel der deutschen Gesellschaft und zugleich normenkongruent durch die Verweisform Wir als Ziel des Schreibers etabliert: ${ }^{13}$

„Wir wollen aber in Deutschland ein gutes und harmonisches Mitei-nander mit den hier lebenden Juden und auch Moslems.“

[ZJD_07.05.2007_Zie_001]

d) Die quasi-vorurteilsfreie Argumentation, bei der die Ablehnung jeglicher Vorurteile betont wird (vgl. hierzu auch Wodak et al. 1990: 353), ist ebenfalls Teil der positiven Selbstdarstellung. In (16) distanziert sich der Textproduzent mitsamt seiner Gruppe explizit von rechter und nationalsozialistischer Gesinnung und verurteilt die Verbrechen des Nationalsozialismus gegenüber den Juden. Kontrastiv zu Anhängern der NS-Zeit, rechtem Gedankengut und somit implizit zum mit diesen Gruppen assoziierten Antisemitismus verortet sich der Textproduzent selbst in der gesellschaftlichen, demokratischen Mitte.

\begin{abstract}
„Ausdrücklich betone ich, dass wir keine 'braune Stammtischbrüder' - sondern ganz normale Deutsche mit einem ausgeprägten Sinn für Gerechtigkeit und guter Demokratie sind. NS-Anhänger sind wir auch keine. Wir verurteilen und bedauern die schlimmen begangenen Taten des NS-Regimes - besonders gegen das jüdische Volk - zutiefst.“ [ZJD_07.05.2007_Zie_001]
\end{abstract}

Über diese Kontrastierung erfolgt die Aufwertung der eigenen Argumentation. Auffallend ist ein emphatischer Sprachgebrauch bezüglich der Modalität der Äußerung. So wird im repräsentativen Sprechakt Festlegen die Sprechereinstellung zum Inhalt der Äußerung mittels des Modalwortes ausdrücklich hervorgehoben. Des Weiteren unterstreicht der Textproduzent durch die Nominalphrase

13 Eine derartige Aussage kann im Textzusammenhang implizieren, dass diejenigen, die nicht in die Wir-Gruppe eingeordnet werden (d. h. die Juden), nicht an einem harmonischen Miteinander interessiert seien und sich somit im Gegensatz zur Wir-Gruppe nicht allgemein gültigen Normen und Werten der Gesellschaft verpflichtet fühlen. 
ganz normale Deutsche und die präpositionale Erweiterung mit einem ausgeprägten Sinn für Gerechtigkeit und guter Demokratie, dass bei ihm die Akzeptanz der staatlichen Basiskonzepte besonders stark ausgeprägt und deshalb keine vorurteilsbehaftete Meinungsäußerung zu befürchten ist. Im expressiven Sprechakt Verurteilen und Bedauern wird mittels des Modaladverbs zutiefst die Dimension der Ablehnung der NS-Verbrechen als besonders intensiv hervorgehoben. Durch diese Angaben legitimiert sich der Schreiber als „quasi-vorurteilsfrei“ (für die dann in der Regel folgenden antisemitischen Äußerungen).

e) Die positive Selbstdarstellung ist weiterhin gekennzeichnet durch das Aufzeigen der eigenen Rationalität ${ }^{14}$ und Objektivität, welches sprachlich oft durch einen sachlichen Stil frei von Emotionsausdrücken sowie durch das Präsentieren von Faktenwissen gekennzeichnet ist, wie in (17):

„Sehr geehrter Herr Spiegel, ich lege Ihnen eine Übersicht über geschichtliche Fakten bei und ich bitte Sie, diese Fakten genau zu beachten.“[ZJD_08.04.2002_Lud_001]

f) Plakativer Philosemitismus, in dem die jüdische Kultur als etwas Besonderes hervorgehoben, bewundert und anerkannt wird, oder ostentativer PhiloIsraelismus, in dem eine bewundernde und anerkennende Haltung gegenüber Israel zum Ausdruck kommt, sind ebenfalls Teil der positiven Selbstdarstellung. Sprachliche Charakteristika solcher Äußerungen sind positiv wertende und oftmals emotionsbezeichnende Lexeme in Verbindung mit philosemitischen Stereotypen, wie in (18), oder ausschließliche Positivattribuierungen in Verbindung mit einem emphatischen Sprachgebrauch, wie (19).

„Ich liebe Kishon und aus tiefstem Herzen Ihre Musik. Vom 'Siebten Kreuz' über sehr, sehr, sehr viele andere Informationen verfügend, empfand ich immer ein Herz für Ihr gequäles Volk !“

[IBD_10.06.2006_Reb_001]

14 Wodak et al. (1990: 353) verwenden den Terminus der pseudorationalen Beurteilung, mit dem gleichzeitig schon die Rationalität der Argumente in Frage gestellt wird. Die Bewertung der Argumentationsbasis hinsichtlich rational oder „nur“ Rationalität vortäuschend sollte aber erst nach der Analyse der Argumente erfolgen. Deshalb plädieren wir für die zunächst neutrale Bezeichnung Aufzeigen der eigenen Rationalität; somit wird die Absicht des Textproduzenten in den Vordergrund gerückt und seine Vorgehensweise beschrieben. 
„Ich habe nach 1947 bis heute eine tiefste Bewunderung für das israelitische Volk empfunden, wie man durch harte Arbeit und hohe Intelligenz das Land ISrael aufgebaut hat.“ [ZJD_09.04.2002_Hor_001]

Solche offensiven Positivbewertungen werden in den Texten immer dann in der Einleitungsphase der Schreiben benutzt, wenn unmittelbar darauf antisemitische Stereotype oder besonders heftige anti-israelische Kritik verbalisiert werden. Die Verfasser wollen sich so gleichzeitig absichern und rechtfertigen.

\subsection{Vermeidungsstrategien und defensive Selbstverteidigung: „Ich bin kein Antisemit!“}

Als Vermeidungsstrategien gelten Argumentationsmuster, mit deren Hilfe die mögliche Brisanz der eigenen Argumentation relativiert wird, also die Gefahr, außerhalb der Political Correctness und/oder der moralisch-gesellschaftlichen Werte sowie Einstellungen der Mitte der Gesellschaft lokalisiert zu werden. Vermeidungsstrategien dienen allgemein der Aufrechterhaltung des positiven Selbstbildes und/oder speziell der direkten und indirekten Antisemitismus-Abwehr und der Abwehr gesellschaftlicher Sanktionen. Zumindest weisen sie darauf hin, dass dem Textproduzenten bewusst ist, dass die Leser die Äußerungen als vorurteilsbehaftet interpretieren können.

Innerhalb der argumentativen Muster zur Aufrechterhaltung des positiven Selbstbildes und der Antisemitismus-Abwehr (s. hierzu die Beispiele (26), (30), (31)) treten einzelne Argumente auf, die ebenfalls in argumentativen Mustern zum Aufbau des positiven Selbstbildes bei den Legitimierungsstrategien zu finden sind (vgl. Beispiele (8), (9), (10)). Der Unterschied liegt jedoch in der Funktion, denn bei den Legitimierungsstrategien dienen die Argumente der positiven Selbstdarstellung, um das Schreiben per se zu legitimieren, wohingegen sie bei den Vermeidungsstrategien dazu dienen, konkrete Inhalte und Äußerungen des Textes dem Antisemitismus-Vorwurf zu entziehen. Legitimierungsstrategien sind somit offensiv, Vermeidungsstrategien dagegen defensiv.

Die Aussage Ich bin kein Antisemit ist (in diversen Varianten) daher bei weitem die am häufigsten verbalisierte Form der Vermeidungsstrategie in den Texten an den ZJD und die IBD. Über 53 Prozent der Schreiber aus der Mitte benutzen sie. Sie wird in verschiedenen Manifestationen entweder am Anfang oder am Ende der Schreiben formuliert, zum Teil auch, insbesondere in langen Texten, mehrmals wiederholt. So soll kein Zweifel daran entstehen, dass der Anlass für die Äußerungen nicht dubios und verwerflich, sondern berechtigt und politisch korrekt ist. 
„Herr Kramer, [...] ich bin NICHT antisemitisch.“

[ZJD_18.05.2005_Ben_001]

(21) „Ich bin garantiert kein Antisemit und verbitte mir Argumentationen in dieser Richtung - meine Kritik ist ausschließlich auf die Politik Israels gerichtet.“[IBD_20.07.2006_Fle_001]

(22) „Ich bin SPD Wähler, schon immer [...] Wie gesagt, Ich als Durschnittsdeutscher äußere Ihnen hier meine Gedanken. Ich bin kein Nazi. Kein Rechtswähler und kein Judenhasser.“ [ZJD_Gaza2009_14/816_ano_001]

Prinzipiell erfüllt die Strategie der Leugnung des eigenen Antisemitismus auch das Kriterium der Selbstlegitimierung. Denn indem der Verfasser negiert, judenfeindlich eingestellt $\mathrm{zu}$ sein, wird impliziert, dass seine heftige Kritik einen anderen, einen legitimen Grund haben muss.

(23) „Ich bin nicht Rechtsradikal, möchte es auch nicht werden. Nur das Verhalten Dieses Gewalttätigen Staates ist abscheulich. Der Staat Israel spielt uns immer vor , was alles im zweiten Weltkrieg passiert ist. Das war auch nicht rechtens, was damals geschah.“ [ZJD_30.07.2006_Bus_001]

(24) „Ich bin kein Nazi! Aber Ihr gieriger Verein mit seinen dauernden Geldforderungen regt mcih auf.“ [ZJD_30.07.2006_Kei_001]

In (23) und (24) antizipieren die Verfasser bewusst den Vorwurf, der ihnen gemacht werden kann. Durch die Vorwegnahme und Negierung des Vorwurfs versuchen sie, ihm seine Berechtigung zu nehmen. Schreiben, die mit solchen Leugnungen beginnen, lassen erkennen, dass sich ihre Verfasser der (prinzipiellen) Brisanz ihrer Äußerungen bewusst sind. Zugleich verteidigen sie diese aber als wichtig, richtig und notwendig. Das Bedürfnis, Kritik, Wut und Empörung auszudrücken, ist am Ende größer als die Sorge, als ein von Ressentiments besessener Antisemit wahrgenommen zu werden.

Als Antisemit betrachtet zu werden wäre für gebildete Schreiber sowohl aus der Fremd- als auch aus der Eigenperspektive ein nicht unerhebliches Problem: Ein Antisemit zu sein widerspricht ihrem eigenen Bild; ihr Selbstkonzept ist geprägt von den Konzepten einer modernen, aufgeklärten und toleranten Gesellschaft, die aus den Erfahrungen der Weltkriege und insbesondere aus den Gräueltaten im Holocaust gelernt hat. Es gehört zur Sozialisation der nach 1945 Geborenen, ein „Nie wieder“ als unumstößliches ethisches Gebot anzuerkennen. Ein gebildeter Bürger, der über den Holocaust Bescheid weiß und daher kognitiv die 
Gefahren ressentiment- und vorurteilsgesteuerter Einstellungen kennt, kann sich vor diesem Hintergrund nicht als bekennender Antisemit präsentieren, wenn er von den Mitgliedern der modernen Gesellschaft ernst genommen und (auch von sich selbst) als anständiger Mensch akzeptiert werden will.

Der vor anderen und sich selbst geleugnete Antisemitismus erhält somit das Wunschbild des vorurteilsfreien, verantwortungsbewussten Bürgers aufrecht. Für den wissenschaftlichen Betrachter der Texte stellt sich hier die Frage, inwieweit die Verfasser ihre zum Teil vehement artikulierten Leugnungsstrategien ernsthaft benutzen (können), obgleich sie in ihren Texten ein antisemitisches Stereotyp nach dem anderen artikulieren, ohne selbst $\mathrm{zu}$ bemerken, dass hier ein Widerspruch entsteht. Motiviert ist diese Vorgehensweise zum einen durch die Tabuisierung von öffentlich artikuliertem Antisemitismus seit 1945, d. h. das ostentative Leugnen der judenfeindlichen Einstellung schützt vor befürchteten Sanktionen. Zum anderen hält diese Leugnung das positive Selbstkonzept des Textproduzenten aufrecht. Aus tiefen- und emotionspsychologischer Sicht kann hier natürlich auch vermutet werden, dass bei einigen dieser Schreiber ein massiver Verdrängungsprozess stattgefunden hat. Sie verweigern sich selbst die unter Umständen schmerzhafte Erkenntnis, ressentimentabhängig zu fühlen und vorurteilsbelastet zu denken und leugnen somit das (für alle anderen) Offensichtliche.
„Als Deutscher der kein Antisemit ist frage ich [...]“ [IBD_17.07.2006_ Wei_001]; „Ich bin deutscher Staatsbürger und kein Antisemit, aber [...]“ [IBD_20.02.2009_Wei_001]; „Ich bin kein Faschist und kein Antisemit ganz im Gegenteil, ich habe selbst jüdische Freunde [...]“ [IBD_06.05.2009_Fil_001]

Auf der sprachlichen Ebene kann die Antisemitismus-Abwehr sowohl explizit als auch implizit erfolgen:

Sprachlich realisiert wird die Antisemitismus-Abwehr beispielsweise durch Selbsteinschätzungen, in denen der Textproduzent sich explizit in der verneinenden Form Ich bin nicht $x$ charakterisiert, wobei $x$ durch Antisemit oder Faschist, Rechter usw. ersetzt werden kann und/oder affirmativ formuliert, Ich bin $x$, wobei $x$ durch Linker, Demokrat usw. ersetzt werden kann. All diese Aussagen haben konzeptuell gemeinsam, dass sie mit 'nicht antisemitisch sein' verbunden sind bzw. mit ihnen die Ablehnung von Antisemitismus assoziiert wird. Derartige repräsentative Sprechakte sind häufig negativ evaluierenden expressiven Sprechakten vorangestellt, in denen dann die kritische Einstellung geäußert wird. In (26) und (27) markieren die Textproduzenten diesen Kohärenzbruch sogar explizit über die Kontrajunktion aber (s. hierzu auch Beispiel (45)): 
„ich bin kein Faschist und sehe mich Mitte-Links angesiedelt. Aber Israel KOTZT mich in der letzten Zeit sowas von an [...]“

[IBD_05.02.2006_ano_001]

(27) „PS: Ich bin nicht Judenfeindlich eingestellt , aber sowas ärgert mich doch sehr !“[IBD_09.10.2007_Gro_001]

Eine indirekte Antisemitismus-Abwehr kann über eine Analogie erfolgen, beispielsweise wenn die Konzeptualisierung des deutsch-israelischen Verhältnisses als ein freundschaftliches und gleichberechtigtes zugrunde gelegt und somit Juden und Deutschen eine gemeinsame Gruppenzugehörigkeit zugesprochen wird. In Beispiel (28) erfolgt dies mit Hilfe des Modalverbs dürfen und über die Konstruktion der Analogie (das eigene Verhalten entspräche dem israelischen), sodass die Aussage des Textproduzenten als legitim erscheint:

„Sowas darf man unter freundn sagen, das tut ihr uns gegenüber ja auch, es hat bisher nur selten welcher gemacht.“[IBD_18.02.2006_Bri_001]

Biographische Auskünfte, wie die explizite Altersangabe des Textproduzenten (als „Gnade der späten Geburt“) und der Verweis auf jüdische Freunde/Bekannte oder Verwandte, s. (30), dienen ebenfalls einer impliziten, s. (29), bzw. expliziten, s. (30), Antisemitismus-Abwehr. Hierin spiegelt sich zugleich auch Schuldabwehr.

(29) „Als deutscher Staatsbürger, der erst 1968 geboren wurde“ [IBD_20.07.2011_Vul_001]

(30) „Vileicht denken Sie ich sei Antisemitisch aber das bin ich nicht, auch ich habe jüdisch Verwandtschaft und finde es Scheiße was früher in Deutschland passiert ist.“[ZJD_23.04.2002_Doe_001]

Eine indirekte Antisemitismus-Abwehr liegt vor, wenn sich die Verfasser zur Schuld und moralischen Verantwortung der Deutschen aufgrund der NSGeschichte bekennen, dieses Bekenntnis aber gleichzeitig als Begründung für die Kritik an der israelischen Politik herangezogen wird, wie in (31). In (31) werden gleichzeitig noch der hohe Bildungsgrad und die Berufswahl als weitere Argumente für die Lehre aus der Geschichte (sich gegen Diskriminierung und Verfolgung aufzulehnen) herangezogen. 


\begin{abstract}
„Als Doktorandin der Geschichte und zukünftige Gymnasiallehrerin halte ich es gerade für Deutschland eine besondere Verpflichtung, sich gegen Diskriminierung und Verfolgung zu wenden - gerade auch im Falle des palästinensischen Volkes.“ [ZJD_09.04.2002_Sto_001]
\end{abstract}

Bei diesem argumentativen Muster lautet die Oberprämisse: 'Die deutsche Vergangenheit verpflichtet die Deutschen, Unrecht und Völkermord zu kritisieren'. Die Prämisse lautet: 'Das palästinensische Volk leidet unter Diskriminierung und Verfolgung', sodass sich als Konklusion ergibt: 'Die Deutschen sind verpflichtet, die Diskriminierung und Verfolgung der Palästinenser zu kritisieren'. Es wird vorausgesetzt, dass die israelische Politik verantwortlich für die Diskriminierung und Verfolgung der Palästinenser sei, sodass sich als erweiterte Konklusion ergibt: 'Die Deutschen sind verpflichtet, die israelische Politik gegenüber den Palästinensern zu kritisieren'.

Auf der sprachlichen Ebene erfolgt die Antisemitismus- und Sanktionsabwehr, wenn z.B. explizit eine mögliche antisemitische Lesart präventiv abgelehnt, ausgeschlossen oder negiert wird.

$$
\begin{aligned}
& \text { „[...] damit kein Missverständnis entsteht. Die folgenden Zeilen sollen } \\
& \text { den gestrigen Selbstmordanschlag in Tel Aviv nicht rechtfertigen.“ } \\
& \text { [IBD_18.04.2006_Bor_001] }
\end{aligned}
$$

Die mögliche Implikatur, dass die Äußerungen antisemitisch motiviert sein könnten, wird in (32) expressis verbis über die Modalverbkonstruktion sollen ... nicht vom Textproduzenten verneint.

„Wie ich hoffe, werden Sie diesen Brief nicht als Kritik verstehen, sondern als Zeichen der Anteilnahme und der Wertschätzung.“

[ZJD_25.09.2006_Hip_001]

In (33), der Zuschrift einer Jura-Professorin, wird die präferierte Lesart dem Rezipienten direkt vorgegeben. Antisemitismus-Abwehr kann auch über die Thematisierung einer gesellschaftlichen Diskreditierung oder einer strafrechtlichen Verfolgung (als mögliche Folgen der Äußerungen) ausgedrückt werden. In Beispiel (34) erfolgt dabei zwar eine explizite Sanktionsabwehr, ohne jedoch die Diffamierung an sich zurückzunehmen:

„wenn es nicht strafrechtlich relevant wäre, würde ich Sie fragen wer den verantwortlichen israelischen Politikern, welche die laufenden Kriegsverbrechen in Gang gesetzt haben, ins Resthirn geschissen hat. Da ich 
mir aber ein Strafverfahren ersparen möchte, richte ich diese Frage nicht an Sie, sondern stelle mir diese selber.“[IBD_02.09.2005_Lur_001]

Es wird eine Konjunktivkonstruktion gewählt, um nicht explizit auf die Proposition festgelegt werden zu können.

Alle diese Formen der Sanktionsabwehr sind metakommunikativ, d. h. die Schreiber kommentieren ihre eigenen Äußerungen und thematisieren mögliche Les- und Interpretationsarten. In dem offensichtlichen Bedürfnis, nicht missverstanden zu werden, kommt ein vorhandenes Problembewusstsein zum Ausdruck: Die Sprecher scheinen sich darüber im Klaren zu sein, dass ihre kommunizierte Einstellung gesellschaftlich oder politisch unter Umständen inakzeptabel ist.

Vermeidungsstrategien, die den brisanten Inhalt des Artikulierten zumindest formal reduzieren sollen und gleichzeitig der Erhaltung des positiven Selbstbildes dienen, erfolgen auf der sprachlichen Ebene über:

a) Abschwächung, ${ }^{15}$ wobei mit Hilfe von expressiven Sprechakten wie Entschuldigungen oftmals in Verbindung mit Modalwörtern (wie leider) die Brisanz der Proposition ${ }^{16}$ gemildert wird bzw. reduziert werden soll, ohne jedoch die Proposition selbst infrage zu stellen:

„Entschuldigung, ich habe ein paar für mich sehr wichtige Fragen!“ [IBD_13.06.2007_Fis_001]

(36) „leider muss ich sagen, so langsam aber sicher versteht man , auch als deutscher, warum es immer wieder Menschen gibt die es schaffen Massen hinter sich zu bringen um gegen das Judentum vorzugehen . Leider muß ich auch sagen , ihr seid das einzige Volk in der ganzen Bibelgeschichte das ständig und immer wieder von der ganzen Welt gehasst wird.“[ZJD_27.07.2006_Zaj_001]

15 Reisigl/Wodak (2001) beschreiben die Abschwächung (bei innen Mitigation) als eine allgemeine Strategie innerhalb der antisemitischen Sprachverwendung, bei der es ihnen zufolge zu einer Abschwächung der eigenen Aussage kommt (vgl. Reisigl/Wodak 2001: 44 f.). Es kommt aber nicht wirklich zu einer Abschwächung der Aussage. Nur formal täuschen die Verfasser Rücksichtnahme auf den Adressaten vor.

16 Es handelt sich hierbei um die Konstruktion einer Doppelproposition EP (P). Eine Aussage erscheint so eingebettet in eine Einstellungsbeschreibung. S. Schwarz-Friesel (2007: 174 f.). 
Die abschwächende Funktion erfolgt über die Semantik der emotionsausdrückenden Wörter und Phrasen, da diese das Gefühl des Bedauerns verbalisieren und zugleich die Einhaltung formaler Höflichkeitskonventionen signalisieren.

In Beispiel (37), von einem promovierten Soziologen an die IBD geschickt, werden der NS-Vergleich (dessen politische Brisanz dem Verfasser wohl bewusst ist) und die moralische Diskreditierung der israelischen Bevölkerung mittels diverser sprachlicher Mittel (Modalpartikel bitte, Emotionsausdruck besorgt, Abschwächung durch die Quasi-Entschuldigung sehen Sie mir ... nach) formal entradikalisiert. Semantisch jedoch bleibt die unangemessene Analogie erhalten.

$$
\begin{aligned}
& \text { „Das ist ein Verbrechen und - sehen Sie mir bitte diesen Vergleich nach } \\
& \text { - diese Art von Attacken erinnern mich leider immer mehr an das Vorge- } \\
& \text { hen der deutschen Nazis gegen die jüdische Bevölkerung in den Ghettos! } \\
& \text { [...] Ich hoffe sehr, die israelischen Entscheidungsträger und die Bevöl- } \\
& \text { kerung wachsen hinsichtlich moralischer Reife. [...] Mit entsetzten und } \\
& \text { äußerst besorgten Grüßen Shalom“ [IBD_12.06.2006_Goe_001] }
\end{aligned}
$$

Zugleich erfolgt eine sprachliche Umdeutung der tatsächlich vollzogenen Sprachhandlung diffamierende Kritik, die stattdessen als besorgte Anteilnahme präsentiert wird. So stellt sich der Verfasser auf der formalen Kommunikationsebene als moralisch integre Persönlichkeit dar. Der ausgeprägte Hang zur Selbstgerechtigkeit, der in dieser E-Mail zum Vorschein kommt, ist allgemein ein typisches Charakteristikum der Schreiber aus der Mitte (s. hierzu Kap. 10.2).

b) rhetorische Fragen, wie in (38), und Konjunktivkonstruktionen, wie in (39), um das explizite Äußern und damit die Festlegung auf einen Äußerungsinhalt zu vermeiden.

(38) „Ist Israel denn gegenüber Deutschland aufgrund der deutschen Vergangenehit unantastbar?“ [ZJD_08.04.2002_Nie_001]

(39) „Sollten sich durch die auch international geäusserte Kritik hieran Juden in aller Welt diskriminiert fühlen, wären Klagen darüber m.E. an die Adresse Israels zu richten und nicht an die Berichterstatter.“ [ZJD_11.08.2006_Bla_001]

Auch hier ist es lediglich die Form der Äußerung, die vorsichtig gewählt ist, doch der kommunikative Sinn ergibt sich unmissverständlich aus den Vorwürfen. 
c) Einschränkung der Diskriminierung mittels Quantoren, wie in (40), und/oder eine kontrastive Einteilung in „böse“ versus „gute“ Juden und/oder Israelis, wie in (41). Insbesondere in Bezug auf Israel differenzieren die Schreiber häufig zwischen der korrupten, verbrecherischen Regierung und der guten, leidenden Zivilbevölkerung, s. (42). ${ }^{17}$

(40) „[...] und bin der festen Überzeugung, dass es auch in Israel viele Menschen gibt, die mit dem was Herr Sharon treibt, nicht einverstanden sind. Schließlich haben nicht alle Israelis diesen Menschen gewählt und manche, die ihn gewählt haben, tut es heute sicher schon leid.“ [ZJD_10.04.2002_Kem_001]

(41) „Es ist immer nur eine Minderheit, die den Konflikt schürt auch die Politik in Israel trägt dazu bei, das der Konflikt immer wieder eskaliert.“ [IBD_18.02.2006_Bri_001]

(42) „Ich bemitleide die israelische Bevölkerung für ihre Regierung, die mit aller Macht dafür sorgt, daß dieser Staat immer weniger Freunde auf der Welt hat.“[ZJD_10.04.2002_Rot_001]

Es kommt auch vor, dass diese Kontrastierung zwischen den Gruppen der „(guten) Holocaustopfer“ und der „(bösen) israelischen Täter“ vorgenommen wird, wie in (43):

(43) „Die Verknüpfung der Verbrechen der gegenwärtigen israel. Regierung mit dem Mord an Millionen Juden durch die Faschisten betrachte ich als eine unerhörte Schändung des Ansehens Ihrer jüdischen Glaubensgenossen, die den Hitlerterror zum Opfer gefallen sind. Scharon ist ein Mörder und rassistischer Kriegstreiber. Er ist ein willfähriger Vollstrecker der Politik der USA. Terroristen sind nicht die Palästinenser, sondern die isreal. Militärs und Siedler, die geraubtes Land besetzt halten.“ [ZJD_14.04.2002_ano_004]

Die jeweiligen Einschränkungen sollen belegen, dass die Schreiber nicht generell alle Israelis und alle Juden verurteilen, sondern nur die „bösen“. Es ist eine

17 Auf diese Weise lassen sich Tatbestände wie z. B. die Raketenschüsse auf Südisrael, die nicht in das eigene Weltbild passen, relativieren. So seien diese Angriffe auf die Zivilisten natürlich zu verurteilen, aber man dürfe eben nicht die Verbrechen der israelischen Besatzungsarmee vergessen, die wesentlich schwerwiegender seien. 
Strategie, die letztlich dem Vorwurf, generell judenfeindlich eingestellt zu sein, entgegentritt.

d) Eine besondere Form der Vermeidungsstrategien bilden Ja-Aber-Konstruktionen. Sie dienen in erster Linie der thematischen Informationsentfaltung im Text und schließen häufig an die Konstruktion eines positiven Selbstbildes an. JaAber-Konstruktionen, die eine Verknüpfung der positiven Selbstdarstellung mit einer vorurteilsbehafteten oder als solcher interpretierbaren Äußerung darstellen, werden zur Aufrechterhaltung des positiven Selbstbildes sowie zur Antisemitismus- und Sanktionsabwehr eingesetzt.

Auf der sprachlichen Ebene wird mittels der Ja-Aber-Konstruktion ${ }^{18}$ der Übergang von der positiven Selbstdarstellung (unter Aufrechterhaltung des positiven Selbstbildes) zur vorurteilsbehafteten Äußerung explizit markiert.

In den Beispielen, in denen die Textproduzenten sich als demokratisch, verantwortungsbewusst und moralisch beschreiben, werden diese gesellschaftlich als positiv bewerteten Eigenschaften als Schreibanlass/Vorwand instrumentalisiert, um sich kritisch zu äußern. Als allgemeines Muster gilt dabei: Eben weil ich $x$ bin, muss ich Folgendes sagen, wobei $x$ mit den oben genannten Selbstattribuierungen gefüllt werden kann.

Der Textproduzent markiert diesen Übergang sprachlich explizit beispielsweise über adversative Konjunktionen oder kausale Adverbien, s. (44), die ein konzessives Verhältnis anzeigen, also einen (scheinbaren) Kohärenzbruch, s. (45), indizieren.

(44) „Diese unfassbaren Ereignisse [Verbrechen in den KZs, d. Verf.] dürfen nicht vergessen oder als erledigt betrachtet werden. Ich kann es aber nicht mehr ertragen, daß die Herren Spiegel und Friedmann ständig mit erhobenen Zeigefinger vor den Deutschen herumfuchteln und sich als Moralapostel aufspielen.“[ZJD_08.06.2002_Her_001]

18 Die argumentativen Muster von van Dijk (2002) folgen auch dem Muster der vorgeblichen, generellen Zustimmung, die dann jedoch aufgeteilt oder eingeschränkt bzw. sogar widerrufen wird. In der Regel folgen solche Aussagen dem Muster Ich habe/bin/denke ..., aber ... und lassen sich auf Antisemitismus und Anti-Israelismus wie folgt übertragen: Scheinbare Leugnung: Wir haben nichts gegen Juden, aber ...; Scheinbare Konzession: Einige von ihnen sind nett, aber im Allgemeinen ...; Scheinbare Empathie: Natürlich haben die Israelis Probleme, aber ...; Scheinbare Ahnungslosigkeit: Ich hab keine Ahnung, aber ...; Scheinbare Entschuldigung: Entschuldigen Sie, aber ...; Rückbelastung (das Opfer bloßstellen): Nicht Sie, wir sind die wahren Opfer ...; Transfer: Mich stört es nicht, aber meine Freunde und Bekannten ... 
„Ich bin kein Antisemit, und trotzdem bin ich der Meinung, der Zentralrat soll sich soweit wie möglich aus der Politik heraushalten.“ [ZJD_20.09.2006_Glo_001]

Der Wechsel von der positiven Selbstdarstellung bzw. normresistenten Evaluation zu einer vorurteilsbehafteten oder als solcher interpretierbaren Äußerung kann aber auch über die Konstruktion einer (scheinbar) kausalen Beziehung mittels konsekutiver Konjunktionen oder Kausaladverbien erfolgen, sodass ein expliziter Kohärenzbruch vermieden wird, wie in (46) und (47).

(46) „Um so mehr missbillige und verurteile ich die derzeitigen Aktionen [...]“ [ZJD_11.08.2009_Man_001]

(47) „ich bin an und für sich ein relativ unpolitischer Mensch, wenn man von der Tatsache absieht, dass ich ein eingefleischter 'Anti-Nazi' bin und dieses auch öffentlich in vieler Form kundtue. Gerade aufgrund dieser Tatsache möchte ich nun doch einmal eine ernsthafte Kritik an Ihrem Umgang mit kritischen Äußerungen zum Staat Israel loswerden.“ [ZJD_14.09.2006_Man_001]

Unter die Strategie der Vermeidung fallen prinzipiell alle impliziten Formen des Verbal-Antisemitismus (vgl. b); s. hierzu bereits Kap. 3). Sie besteht darin, dass sprachliche Äußerungen, die eine bestimmte Form-Funktion-Kopplung (z.B. Aussagesatz-Feststellung) haben, mit abweichender Funktion benutzt werden. Diese Äußerungen folgen dem Schema: Der Sprecher sagt x, meint aber tatsächlich y.

Die Äußerung (48), von einem promovierten Mediziner an den Zentralrat gesendet, scheint auf den ersten Blick ein reiner Assertiv zu sein, d. h. ein Sprechakt, mit dem der Sprecher eine Aussage über die Welt trifft bzw. sich auf einen Wahrheitswert festlegt, also ganz allgemein eine überprüfbare Behauptung oder Aussage verbalisiert.

„Gewalt wird immer neue Gewalt nach sich ziehen, wenn es kein Innehalten, keine Besinnung gibt.“[ZJD_07.04.2002_Bin_001]

(48) als Assertiv zu verstehen, erklärt jedoch nicht den kommunikativen Sinn: Warum sollte der Mediziner den Zentralrat der Juden über den Allgemeinplatz „Gewalt zieht Gewalt nach sich“ informieren wollen? Diese Information ist für den Zentralrat nicht neu. Die kommunikative Absicht liegt hier nicht in einer Aufklärung, sondern vielmehr in einer Aufforderung zum Innehalten bzw. Einhalten. 
Beispiel (48) kann also als Sprechakt mit direktivem Handlungswert (Auffordern) beschrieben werden, obwohl über die wörtliche Bedeutung nur eine Feststellung ausgedrückt wird. Gleichzeitig vollzieht sich hier die konzeptuelle Gleichsetzung von deutsch-jüdischen und israelischen Belangen, und der Verfasser stigmatisiert den Zentralrat als Mittäter bzw. mitverantwortliche Instanz für die Gewalt in Israel (s. Kap. 7.2.1 und 5.2).

Viele Aussagen werden mit den performativen Verben sagen, betonen oder feststellen realisiert und enthalten Abschwächungsmittel wie die Modalpartikeln bitte, leider etc.

„Leider muss ich ihnen nunmehr sagen das ich nicht mehr als Verachtung für diese primitive Art der 'Poltik' habe.“[ZJD_10.08.2006_Koe_001]

Tatsächlich handelt es sich aber um Verbal-Angriffe, die die Adressaten diskriminieren und diffamieren. Die kommunikativen Absichten der Schreiber, also Beleidigen oder Drohen, werden so entschärft, Diffamierungen und Beschimpfungen entsprechend als „,berechtigte Kritik“ oder in (50) gar als „Mitgefühl“ deklariert dargeboten.

„werten Sie dieses Schreiben als Zeichen des Mitgefühls für ihr Volk.“ [IBD_23.07.2006_Vol_001]

Sprechakte wie Sagen und Feststellen haben normalerweise die Funktion, den Wahrheitswert des ausgedrückten Inhalts festzulegen. Bei den Zuschriften an den ZJD und die IBD drücken diese Sprachhandlungen dagegen Beleidigungen, Diffamierungen und/oder subjektive Einschätzungen aus (s. hierzu Kap. 10).

Die diskriminierende und ressentimentgeladene Argumentation wird zumeist verteidigt als „legitime Israel-Kritik“ (wobei der Vorwurf des Antisemitismus antizipiert und zurückgewiesen wird. Zur Abgrenzung von Anti-Israelismus und Israel-Kritik vgl. Kap. 7.1):

$$
\begin{aligned}
& \text { „Nun bitte nicht mit den alten Klischees argumentieren, wer Israel kri- } \\
& \text { tisiert, wer Israel eine Mörderregierung bescheinigt, ist ein Antisemit.“ } \\
& \text { [ZJD_06.04.2002_5] }
\end{aligned}
$$

Die kommunikative Strategie, Israel anzugreifen, hat für die Schreiber den Vorteil, Schuld zu projizieren und Täterschaft auf der Adressatenseite zu konstruieren, ohne sich dem Verdacht auszusetzen, ein Antisemit zu sein (s. hierzu auch die Täter-Opfer-Umkehrung). Auf Israel wird verwiesen, tatsächlich aber geht es auf einem verbalen Umweg gegen Juden und Judentum. 
Entsprechend verhält es sich mit den Direktiva (also Handlungsaufforderungen), wobei es jedoch quantitativ einen Unterschied zwischen den Zusendungen an den Zentralrat der Juden in Deutschland und an die Israelische Botschaft gibt; bei letzterer treten Direktiva weitaus seltener auf. Unabhängig vom Empfänger enthält die Mehrheit aller direktiven Sprechakte Verben im Imperativ, es handelt sich also um klassische Aufforderungssätze.

$$
\begin{aligned}
& \text { „Stoppt eure Brüder in Israel und macht endlich Frieden!““ } \\
& \text { [ZJD_30.07.2006_Mra_001] }
\end{aligned}
$$
„walten Sie der Gerechtigkeit, damit diese Verbrechen [,ethnischen Säu- berung von Palästina“, d. Verf.] gesühnt werden und endlich Frieden einkehren kann - ein Frieden, den Sie, wir und die ganze Region um Israel braucht. Frieden und nicht immer wieder Hass schüren.“ [ZJD_03.12.2008_Oet_001]

Solche Direktiva vermitteln implizit stets Negativbewertungen (wie Sie besetzen unrechtmäßig das Land anderer oder Bislang setzt sich der Zentralrat nicht für Frieden ein o. Ä.). Die im Korpus vorkommenden Expressiva, ${ }^{19}$ also Sprachhandlungen, die die emotionale Einstellung des Schreibers ausdrücken, werden häufig mit performativen Verben, vor allem protestieren, verurteilen und (be)danken, getätigt oder über konventionalisierte Floskeln (wie es ist eine Schande, es ist unertragbar, dass $x$ ) realisiert. So werden Schreiben oft wie in Beispiel (54) eröffnet:

$$
\begin{aligned}
& \text { „ich protestiere nachdrücklich und entschieden gegen die fortgesetzten } \\
& \text { und vorsätzlichen Vergehen gegen die Menschenrechte [...]. Es ist eine } \\
& \text { Schande, durch nichts zu rechtfertigen. Israel stellt sich nach meiner } \\
& \text { Ansicht dadurch in die Reihe der Schurkenstaaten.“ } \\
& \text { [IBD_29.03.2004_Sta_001] }
\end{aligned}
$$

Werden Kommissiva, also Sprechakte, mit denen sich der Sprecher auf eine zukünftige Handlung festlegt, verbalisiert, sind sie meist als Drohungen zu ver-

19 Die klassische Sprechakteinteilung sieht fast ausschließlich die Expressiva als Möglichkeit, Gefühle sprachlich auszudrücken bzw. Äußerungen mit emotionalem Gehalt zu tätigen. Letztlich können wir aber mit allen Sprachhandlungstypen emotionale Zustände und Prozesse ansprechen und auslösen. Jede sprachliche Äußerung kann emotionale Inhalte vermitteln (s. Schwarz-Friesel 2007: 27). 
stehen. Explizit taucht dieser Sprechakttyp z. B. in Form von Boykottankündigungen wie in Beispiel (55) auf.
„Ab sofort werde ich keinerlei Waren mehr kaufen, die aus Ihrem Land kommen! Und ich werde alle meine Bekannten und Freunde auffordern es genauso zu machen [...]“ [IBD_13.07.2006_Fis_003]

Vordergründig wird auch in (56) nur die Aussage getroffen, Ariel Scharon sei ein Monster, jedoch wird auch hier zugleich ein zweiter Handlungstyp (Expressiv) vollzogen, da die Aussage die Einstellung des Schreibers beinhaltet.

„Ein Monstrum ist er [Ariel Scharon, d. Verf.] und kein Regierungschef.“ [ZJD_10.04.2002_Alt_001]

Dies geschieht über die Auswahl des pejorativen und dehumanisierenden Lexems Monstrum. Gleichzeitig wird damit auch der Akt des Beleidigens vollzogen.

Zusammenfassend lassen sich zwei Typen impliziter Verbal-Antisemitismen bei der Vermeidungsstrategie unterscheiden: Das Gesagte wird entweder mittels reklassifizierender Sprachformen ausgedrückt, umgedeutet und aufgewertet, wodurch das tatsächlich Gemeinte scheinbar negiert wird, oder das Gemeinte wird über zusätzlich zu ziehende Schlussfolgerungen (Implikaturen) vermittelt. Der erste Typ lässt sich paraphrasieren durch 'Das Gemeinte ist etwas Anderes als das Gesagte'; der zweite Typ als 'Das Gemeinte ist etwas Zusätzliches zum Gesagten'.

Das tatsächlich Gemeinte indirekt zu verbalisieren bzw. umzubenennen, hat für den Schreiber stets den Vorteil, sich nicht festlegen zu müssen; er kann sich jederzeit auf das expressis verbis Gesagte beziehen und das Gemeinte, implizit Vermittelte, leugnen. Bei allen indirekten Sprachhandlungen und allen Formen von Vermeidungsstrategien handelt es sich um eine Verschlüsselung judenfeindlicher Vorurteile, die es den Schreibern erlaubt, eine radikale Semantik über eine entradikalisierte Form zu vermitteln.

\subsection{Rechtfertigungsstrategien: „Sie provozieren das!“}

$\mathrm{Zu}$ den Rechtfertigungsstrategien zählen Begründungsmuster, mit denen die eigene Argumentation als rational und begründet abgesichert bzw. bewiesen werden soll. Relevant sind solche Begründungen, wenn eine persönliche und/ 
oder gesellschaftliche (moralische) Schuld bzw. Verantwortung der Deutschen gegenüber Juden und/oder Israel zurückgewiesen und delegitimiert wird. ${ }^{20}$

Den Kern der Rechtfertigungsstrategien bilden Argumentationsmuster, mit denen aufgezeigt wird, dass die vorgetragene Argumentation, die Verknüpfung der einzelnen Argumente, inhaltlich richtig, gesellschaftlich akzeptiert oder verbreitet ist. Es werden Motive genannt, und diese werden als „faktenbasiert“ ausgegeben. Es werden vermeintlich faktische Argumente angeführt, die allerdings de facto subjektiv sind. Es sind das „Schweigen“ und das „moralische Versagen des Zentralrats“, die „Unrechtspolitik“ und der „Staatsterror Israels“, die die Verfasser an den Schreibtisch bzw. PC treiben:

„Ich muss Ihnen schreiben, weil ich mir große Sorgen um den Weltfrieden und um die Zukunft unserer Kinder mache.“[ZJD_28.07.2007_Gab_001]

„Und ich sage: die jetzt den Mund nicht aufmachen gegen diese Verbrechen, gegen diesen Horror, machen sich durch ihr Schweigen automatisch mitschuldig.“ [ZJD_30.08.2006_Cra_001]

„Mittlerweile bin ich so sehr enttäuscht von Ihrer jahrelangen einseitigen neg. Darstellung der Palästinenser und Ihren vielen Provokationen, Ihrer fehlenden Einsicht, Ihrer provokativen Siedlungspolitik, Ihrem Eklat mit der Türkei usw.“ [IBD_01.11.2011_Ber_001]

Über die Legitimations- und Vermeidungsstrategien hinausgehend (bei denen die Legitimierung des Schreibens an sich und die Rechtfertigung einzelner Äußerungen/Argumente im Vordergrund stehen) trägt die Rechtfertigungsstrategie nicht nur zur Aufrechterhaltung des positiven Selbstbildes und insbesondere zur Antisemitismus-Abwehr und historischen Entlastung bei. Vielmehr wird hier neben der Betonung des globalen Anliegens (Sorge um den Weltfrieden, Verantwortungsbewusstsein, Normalität für Deutsche usw.) zusätzlich die Diskreditierung der Adressaten in den Vordergrund gerückt und der Eindruck von Faktizität und Objektivität vermittelt. Zugleich manifestieren sich bei den Rechtfertigungsversuchen der Schreiber oft Schuld- und Verantwortungsabwehr. Diese erfolgen

20 Gleichzeitig handelt es sich bei dieser Abwehr um eine Gegenposition zum offiziellen gesellschaftlichen Konsens des politischen Mainstreams, der die Singularität des Holocaust, die deutsche Schuld und die heutige Verantwortung gegenüber Israel anerkennt. Hier spiegelt sich also ein Konflikt zur Normen-Akzeptanz wider, den die Schreiber für sich jedoch dadurch (auf)lösen, dass sie sich als mutige Verfechter von Meinungsfreiheit präsentieren, die sich nicht dem unterstellten Meinungsdiktat der Juden beugen. 
über argumentative Muster, die eine Rationalität/Kausalität der Einstellung konstruieren oder die eigene Position als gesellschaftlich akzeptiert und mehrheitlich verbreitet darstellen. Die folgenden Beispiele werden aufgrund der Komplexität ihrer Begründungsmuster und der zugrunde liegenden Konzeptualisierungen teilweise etwas ausführlicher diskutiert.

a) Über die Konstruktion einer Analogie werden im folgenden Beispiel (60) jüdische und nicht-jüdische Deutsche gleichermaßen als Opfer und Leidtragende (einer Schicksalsgemeinschaft) des Nationalsozialismus dargestellt. Aufgrund dieser Aufrechnung und des Vergleichens von Leid werden das Ausmaß der nationalsozialistischen Verbrechen an der jüdischen Gemeinschaft und eine persönliche Schuld der damaligen Generation von nicht-jüdischen Deutschen negiert, um so die heutige deutsche Verantwortung gegenüber der jüdischen Gemeinschaft in Abrede stellen zu können (vgl. hierzu auch die Relativierungsstrategien).

„Auch mein Großvater ist im Krieg gefallen und mein Vater mußte für die Mutter, und 3 kleine Geschwister sorgen. Wenn der Großvater sich damals geweigert hätte, dann hätten sie ihn erschossen. Also was wollen Sie eigentlich?“[ZJD_01.08.2006_Mue_001]

Auf der sprachlichen Ebene wird die Analogie mittels der additiven Junktion auch sowie über die kausalitätsanzeigende Wenn-dann-Satzkonstruktion realisiert. In der rhetorischen Frage wird schließlich als Resümee die Konsequenz aus der konstruierten Analogie gezogen: 'Juden haben keinen Grund zu klagen'. Der Vergleich basiert auf dem Argument 'Deutsche haben in der NS-Zeit genauso gelitten wie Juden'. Bezüglich der Deutschen findet anhand eines konkreten familiären Einzelschicksals eine Generalisierung statt, bei der die Beteiligten als repräsentativ für die Gesamtheit der Deutschen stehen. Zugleich bleibt die Analogie vage, denn die jüdischen Opfer werden weder als solche explizit benannt noch wird die Art ihres Leidens beschrieben, lediglich der Zentralrat wird durch das Anredepronomen Sie persönlich als Vertreter der jüdischen Gemeinschaft angesprochen.

b) Über deduktives Schließen wird in Beispiel (61) mit Hilfe eines indirekten NSVergleichs die Rationalität der dargelegten Haltung zu belegen versucht:

(61) „Zu Recht machen die Juden den Deutschen zum Vorwurf, während des Nazi-Regimes aus Feigheit weggeschaut zu haben. Jetzt verlangen Sie jedoch von uns erneut, wegzuschauen. Dazu bin ich aber nicht bereit.“ [ZJD_01.08.2006_Fle_001] 
Im ersten assertiven Sprechakt werden die Deutschen mit dem negativ bewertenden Attribut feige charakterisiert, und diese amoralische Eigenschaft wird als Ursache für die Mitschuld der deutschen Gesellschaft an den NS-Verbrechen evaluiert. Zunächst wird also ein moralisches Versagen der Deutschen explizit eingestanden. In der anschließenden Behauptung wird dem Zentralrat unterstellt, er verlange jetzt erneut von den Deutschen, wegzuschauen, also wiederum moralisch zu versagen. Diese Äußerung impliziert durch das Verb verlangen und die adverbiale Bestimmung erneut erstens, dass das Wegschauen in der NS-Zeit gefordert, also keine freiwillige Entscheidung war, und zweitens, dass die jetzige Situation in Israel mit der NS-Zeit vergleichbar ist, sowie drittens, dass die israelische Politik dem Zentralrat zufolge nicht kritisiert werden darf. Der Textproduzent lehnt dann das als amoralisch diskreditierte Verhalten des Wegschauens $\mathrm{ab}$, denn dieses führe, wie die deutsche Geschichte zeige, zu einem berechtigten Vorwurf. Die Einstellung des Autors gegenüber dem kritisierten Zentralrat wird somit über das deduktive Schließen und den indirekten NS-Vergleich als eine rational und moralisch aus der Geschichte abzuleitende Haltung gerechtfertigt.

c) Der Verweis auf Andere ist ebenfalls als Rechtfertigungsstrategie zu sehen, wenngleich diese auch stark an die Funktion der Vermeidung gekoppelt ist (da der Schreiber es vermeidet, die vorgetragene Meinung als seine eigene auszudrücken). Eine antisemitische Äußerung wird dabei nicht als persönliche Meinung, sondern auf der sprachlichen Ebene explizit als Einstellung einer anderen Person oder Gruppe wiedergegeben, wie in (62):

$$
\begin{aligned}
& \text { „Hier hat man den Eindruck die Juden fühlen sich wohler, je mehr Feinde } \\
& \text { sie um sich haben. Und ich spreche hier nicht von mir, sondern das ist die } \\
& \text { Reaktion aus meinem Bekanntenkreis. Diese, sonst gemäßigte Rechts- } \\
& \text { und auch Linkswähler, bekommen in der Diskussion über dieses Thema } \\
& \text { richtig extremistische Gedanken.“[ZJD_24.03.2005_Dei_001] }
\end{aligned}
$$

Der Produzent lehnt einerseits die Verantwortung für die antisemitische Aussage (die das Stereotyp des STREIT- UND RACHsüCHTIGEN JUDEN bedient) ab, indem er sie einer anderen Instanz zuweist, zugleich aber die wiedergegebene Aussage nicht ablehnt und damit implizit akzeptiert. Andererseits rechtfertigt und legitimiert er durch den Hinweis, dass eine ganze Gruppe von integren Bürgern so denke, das zuvor Geäußerte. Der Verfasser von (63) präsentiert auf diese Weise (zugleich die Abschwächungsstrategie mittels der Verwendung von leider benutzend und sich damit distanzierend) die angeblich mehrheitsfähige Antipathie gegenüber dem Zentralrat der Juden. 
„Durch meine Außendiensttätigkeit in den Bundesländern Hessen, Rheinland - Pfalz und Saarland erfahre ich täglich mehrrnals die Meinungen von vielen Bürgerinnen und Bürgern unseres Vaterlandes, [...]. Nur kann und muss ich leider (!) feststellen, daß die überwiegenden Sympathien in dem immer schärfer werdenden Schlagabtausch zwischen Friedmann und Möllemann, sich auf der Seite von Herrn Möllemann - ganz eindeutig ! - befinden.“[ZJD_11.06.2002_Win_001]

Neben der exklusiven Variante wie in (62) und (63) findet sich auch oft die Strategie der inklusiven (den Schreiber mit einbeziehenden) Meinungsgeneralisierung. Sie dient der Absicherung, dass die negative Haltung nichts Exzeptionelles, sondern vielmehr eine von vielen Deutschen getragene, normale Einstellung sei:

(64) „In meinem Freundeskreis hat sich das Denken über Israel in den letzten 5 Jahren so nachhaltig zum Schlechten verändert, dass wir uns selbst wundern. Nein, wir sind keine Antisemititen. Wir sind in der Regel überdurchschnittlich Gebildete, links orientierte und humanistisch geprägte Deutsche mittleren Alters.“[IBD_08.07.2011_Wac_001]

d) Das Sprechen im Namen der Mehrheit ${ }^{21}$ wird somit als Ausdruck für die allgemeingültige Akzeptanz und Verbreitung der dargelegten Einstellung verwendet:

„Ein Bundesbürger“ [IBD_24.03.2004_ano_002] $]^{22}$

(66) „Es grüßt Sie ein Mitglied der 'unanständigen Mehrheit' die Israels Machenschaften als völkerrechtswidrig ansieht."

[IBD_23.07.2004_Sch_001]

(67) „Mit Gruß einer der 65\% Deutscher, die im Staat Israel eine Bedrohung des Weltfriedens sehen [...]“ [IBD_25.03.2004_War_001]

Auf der sprachlichen Ebene sind hierbei die Selbstreferenzen der Produzenten in der 3. Person Singular sowie die Unterspezifikation auffällig. Oftmals stellt sich der Autor in der den Brief abschließenden Grußformel als Teil eines Kollek-

21 Siehe hierzu auch Wir-Gruppe-Ihr-Gruppe-(In-Group-Out-Group-)Konstruktionen.

22 Beispiele wie (65) können auch eine Form der Anonymisierung darstellen, wenn sich der Absender in der gesamten Zuschrift (Umschlag, Kopfbogen usw.) namentlich nicht nennt. Akademiker jedoch, die sich als solche zu erkennen geben, teilen immer Namen und Adresse mit. 
tivs dar, wie in (65), welches hinsichtlich seiner Quantität als Mehrheit, s. (66), und seiner Qualität als moralisch integer, s. (67), charakterisiert wird. In diesem Zusammenhang werden oft Lexeme wie Weltfrieden und Völkerrecht verwendet, s. (66) und (67), sowie Komposita oder Derivationen auf Basis dieser Lexeme gebildet, wie in (66). ${ }^{23}$

Das Sprechen im Namen der Mehrheit kann auch durch den expliziten Verweis auf ein (jedoch unterspezifiziert bleibendes) Kollektiv erfolgen, wie in (68):

„Die israelische Führung hat [mit ihrem Verhalten, d. Verf.] nicht nur bei mir das Existenzrecht Isreals in Frage gestellt.“

[IBD_Datum_unleserlich_Brief]

Durch Mehrfachunterschriften, wie in (69), und/oder oder durch das Anhängen von Unterschriftslisten und das Einreichen von Petitionen wird diese Strategie ebenfalls oft realisiert:

$$
\begin{aligned}
& \text { „W. L., Berlin, R. S. , Berlin, H. D., Fürth, A. H., Berlin [usw.]“ } \\
& \text { [ZJD_19.04.2002_Lin_001] }
\end{aligned}
$$

Tritt diese argumentative Generalisierung im Abschlussteil des Textes auf, ergibt sich als zusätzliche kommunikative Funktion der spezifischen Rechtfertigungsstrategie noch eine Rechtfertigung des Gesamttextes.

Indem die Verfasser unter Rekurs auf ein allgemeines Meinungsbild die antisemitischen Inhalte als gesellschaftsfähiges Gedankengut darstellen und als anti-antisemitisch deklarieren, legitimieren sie ihre Radikalität vor den Adressaten und auch vor sich selbst.

e) Der explizite Bezug auf (gesellschaftlich anerkannte) Autoritäten ${ }^{24}$ bzw. Institutionen dient ebenfalls dem Selbstschutz durch Verantwortungsabgabe, wobei gleichzeitig eine allgemein positive Zustimmung zum Inhalt der brisanten Äußerungen suggeriert wird. Der Produzent verortet damit seine Einstellung als gesellschaftlich akzeptiert und legitimiert sich.

23 Gleichzeitig wird über derartige Äußerungen auch Normentreue und positive Selbsteinschätzung signalisiert, sodass hier eine Überschneidung verschiedener Strategien vorliegt.

24 Der Bezug auf Autoritäten ist eine argumentative (und persuasive) Strategie, die bereits in der klassischen Rhetorik genannt wird (vgl. z. B. Klein 1994). 
„Es ist eine Gräueltat, welche als Bestrafung der Bevölkerung Gazas verübt wird. [...] Es ist ein Verbrechen. [...] Ich denke es ist eine Abscheulichkeit, dass dies weiter andauert', sagte der ehemalige amerikanische Präsident Jimmy Carter am Freitag.“ [IBD_21.04.2008_Gel_001]

Bei den argumentativen Begründungszusammenhängen, aber auch bei den Rechtfertigungs- und Legitimierungsstrategien spielen Verweise auf Autoritäten eine herausragende Rolle. Um die eigene Meinung $\mathrm{zu}$ begründen, wird auf die Aussagen von Politikern, Nahostexperten oder Historikern verwiesen. In diesem Zusammenhang werden zusätzlich oft die Kommentare verdienter Persönlichkeiten und Würdenträger zitiert. ${ }^{25}$ Als Autorität gilt derjenige, dessen Status und Integrität allgemeine Achtung findet. Wer sich selbst auf eine solche Autorität berufen kann, profitiert von dieser Integrität; seine Meinung ist dann für andere leichter annehmbar oder zustimmungswürdig.

Solche „Autoritätenbeweise“ treten in israelfeindlichen und israel-kritischen Zuschriften vor allem bei Schreibern der Mitte und bei Linken oder Linksextremen auf.

Bei anti-israelischen Äußerungen ist der Bezug auf UN-Resolutionen eine frequente Strategie des institutionellen Autoritätenbezugs:
„Sie werfen dem Libanon vor, der UNO-Resolution 1559 nicht nachge- kommen zu sein. Gibt es überhaupt eine der unzähligen Resolutionen, der Israel jemals nachgekommen ist ? Da ist doch Heuchelei und Ver- dummung.“[ZJD_21.07.2006_Gie_001]

Die von den Schreibern angeführten „Beweise“ für die „Verkommenheit“ von Israelis und/oder Juden lassen allerdings stets wichtige Informationen aus: z. B., dass bestimmte Beschlüsse der UN zurückgenommen wurden und dass die Resolutionen zur Verurteilung Israels stets von arabischen Mitgliedsstaaten eingereicht werden.

Es werden keineswegs nur anerkannte Autoritäten zitiert: Als Autorität zählt bei manchen Schreibern prinzipiell jede/r, die/der im massenmedialen Diskurs eine gewisse Bekanntheit hat oder durch eine exzessiv israel-kritische Haltung aufgefallen ist.

25 Hier konzentrieren wir uns zunächst auf personale Autoritäten; mediale Autorität in Form von Meinungsführermedien und Mainstream-Berichterstattung werden im Zusammenhang mit dem Phänomen der Intertextualität diskutiert. 
(73) „Die mutige und überzeugende Argumentation von Dr. Norbert Blüm, Ulrich Kienzle und Udo Steinbach hat mich sehr verwundert und tief beeindruckt.“[IBD_22.01.2009_Wei_001]

(74) „Nehmen Sie sich ein Beispiel, Frau Knobloch, an der Tochter Ihres Vorgänges Galinski, Frau Evelyn Hecht-Galinski, die als Aktivistin von 'Jews for a Just Peace' ihre Augen nicht verschließt und dieses Unrecht in ehrenwerter Weise beim Namen nennt, anstatt sich in eine Schafherde einzureihen, die hinter Herrn Olmert hertrottet.“ [ZJD_31.08.2006_Goe_001]

f) Der Bezug auf (jüdische) Autoritäten ist eine (von allen Schreibern, gleich welcher politischen Richtung) frequent benutzte, offensichtlich als ausgesprochen schlagkräftig und wirkungsvoll beurteilte Rechtfertigungsstrategie. Hierbei handelt es sich um die klassische persuasive Strategie des Autoritätenbeweises, bei dem als besonders überzeugendes rhetorisches Mittel ein Vertreter der kritisierten Gruppe zitiert wird, der seine Gemeinschaft selbst kritisiert, um so einen internen Beweis anzubieten.

Insgesamt lassen sich drei Formvarianten unterscheiden, mit denen sich die Autoren strategisch auf jüdische „Autoritäten“ beziehen, um ihre eigene Meinung oder die Interpretation des Geschehens in Nahost argumentativ abzusichern:

1. spezifischer Bezug auf eine konkrete jüdische oder israelische Person,

2. vager Bezug auf jüdische oder israelische Freunde und Bekannte,

3. Kontrastierung „gute“ versus „schlechte“ Juden ohne Bezug auf konkrete Personen (d. h. Generalisierung durch Dichotomisierung).

Die häufigste Variante stellt der explizite Bezug auf individuelle jüdische oder israelische Autoritäten dar; diese werden als gute oder positive Vertreter der Juden in Abgrenzung zu den kritikwürdigen schlechten Juden herangezogen. $\mathrm{Zu}$ den Repräsentanten der schlechten Juden zählen in erster Linie die beiden Institutionen IBD und ZJD, die man mittels Autoritätenbeweis kritisieren will. Daneben werden als Einzelpersonen am häufigsten Charlotte Knobloch, Dieter Graumann, Salomon Korn, Ehud Olmert und Schimon Stein erwähnt. Sind keine spezifischen Einzelpersonen genannt, werden unklar eingegrenzte jüdische und israelische Gruppen als kollektive Vertreter der schlechten Juden/Israelis angeführt, wie in (75):

(75) „in unserer westlichen Hemisphäre gibt es seriöse und schlitzohrige Menschen. Die Juden darf man wohl auch klassifizieren nach 'Falken' und ‘Tauben'.Zu den ‘Tauben' gehören soziale, kluge, gebildete, liebens- 
werte, sensible und und musische Menschen. Zu den 'Falken' gehören aggressive, raffgierige und machtbesessene Menschen - Paul Wolfowitz jüd. Medienmacher der deutschen Presse - israelische Politiker, etc. so zumindest nach meinem Empfinden.“[ZJD_16.04.2007_Wac_001]

Auffällig ist in (75) die explizite kontrastive Attribuierung der beiden Gruppen in „seriös, sozial, klug, liebenswert, sensibel und musisch“ versus „schlitzohrig, aggressiv, raffgierig und machtbesessen“. Hier werden der Gruppe der schlechten Juden zugleich antisemitische Stereotype zugewiesen. Derartig plakative Einteilungen finden sich vor allem bei rechtsradikalen und linksextremistischen Schreibern, aber auch vereinzelt bei Verfassern aus der Mitte.

Die Kontrastierung von „guten“ versus „schlechten“ Juden ohne direkten Bezug auf einzelne Personen tritt vor allem dann auf, wenn Israel pauschal kritisiert wird. Die generische Kollektivierung aller Israelis spricht für die Bildung neuer israel-bezogener Stereotype, denn nicht der einzelne konkrete Politiker oder Soldat wird beschuldigt, sondern der Staat Israel oder die Gruppe der Israelis insgesamt (s. hierzu Kap. 7). Diesen generalisierten schlechten Juden werden die achtenswerten, weil israel-kritischen Juden oder Israelis als positive Ausnahmen gegenübergestellt. Auf deren Haltung zu Israel bzw. ihre zum Teil anti-israelischen Äußerungen und Texte bezieht man sich geflissentlich, um alle anderen „uneinsichtigen“ Juden und Israelis zu diskreditieren. In der Regel werden bekannte linke Israel-Kritiker zitiert, allen voran Uri Avnery, aber auch Tony Judt, Benny Morris, Michael Lerner oder Reuven Moskovitz. Von den deutschen Juden sind Felicia Langer, Rolf Verleger oder Evelyn Hecht-Galinksy die Meistzitierten (vgl. Bsp. (76)). Als israelische moralische Autoritäten werden zudem auch David Grossman, Abraham B. Yehoshua und Amos Oz geführt. Auch Gruppen wie „Jews for a Just Peace“ oder „Bürgerrechtler in Israel“ (vgl. Bsp. (77)) werden zu den guten Juden gezählt.

(76) „Sehr geehrte Frau Knobloch, sehr geehrter Herr Dr. Graumann, ich bin froh, dass es Menschen wie Frau Hecht-Galinski, Frau Wieczorek-Zeul und Dr. Verleger gibt, die deutlich ihre Meinung zu den Vorgängen im Libanon sagen. [...] Vor allem aber ist es zynisch. Wenn Sie die Ministerin derart angreifen. Und wissen Sie, Herr Dr. Graumann, was noch zynisch ist? Dieses (Ihres!) Zitat: 'Die israelischen Streitkräfte bemühen sich nach Kräften, Zivilisten zu schonen und Kolateralschäden zu vermeiden. Gleichwohl gilt es, Raketenstellungen unschädlich zu machen, um die eigene Bevölkerung vor weiteren Angriffen zu schützen.' Mit Streubomben? [...] So bleibt das Ergebnis dieser Untersuchung ab-zuwarten, aber vor allem auch der Dank an die Personen, die ich am Anfang dieser Mail 
genannt hatt- und die ausdrückliche Missbilligung Ihres- Frau Knobloch und Herr Dr. Graumann!-Verhaltens.“ [ZJD_02.09.2006_Fis_001]

(77) „Offensichtlich sind etliche der Israelis mit mher Menschlichkeit gesegnet als dieser Zentralrat! Die Armee kann offensichtlich tun was sie will und ihr klatscht Beifall. Nehmt euch ein Beispiel an den Bürgerrechtlern in Israel. Was soll das nach dem Holocaust? Ihr seit wahrlich grosse Bürger der Menschlichkeit!“ [ZJD_22.11.2006_ano_001]

Der explizite Bezug auf jüdische Autoritäten läuft häufig nach dem gleichen Muster ab. Der Verweis erfolgt am Anfang der Zuschrift. Die herausragende Stellung der Autorität wird unterstrichen, indem beispielsweise akademische Titel angeführt werden oder die Personen attribuiert werden als „renommiert“ oder „bekannt“. Wie im folgenden Beispiel (78) sind der explizite Verweis auf die jüdische Abstammung des zitierten Professors sowie hervorhebende Interpunktionszeichen typisch.

(78) „'...] Israel ist blind gegenüber der Gefahr, dass seine Exzesse bis hin zum Einmarsch in den Libanon seinen imperialen Mentor an den Punkt der Irritation und darüber hinaus bringen. [...] radikaler Wandel [in der Politik Israels, d. Verf.] würde freilich jedes Klischee und jede Illusion in Frage stellen, mit denen sich Israel und seine politische Elite so behaglich eingerichtet haben.' Tony Judt, (jüdischer!) Prof. an der Universität New York““ [IBD_20.07.2006_Hau_001; Hervorhebung im Original]

Auffällig sind Modalpartikeln wie sogar, selbst oder auch, die implizieren, dass jüdische Kritik an Israel irgendwie bemerkenswert sei oder besondere Aufmerksamkeit verdiene (vgl. Bsp. (79)).

(79) „Man kann die Parteilichkeit der Frau Knobloch als Jüdin verstehen, aber nicht hinnehmen. Sie soll doch etwas im Rahmen bleiben. Die israelische Armee hat schon von Anfang an brutale Angriffskriege geführt. Die bestätigte sogar der israelische Historiker Benny Morris.“ [ZJD_25.07.2006_Wie_001]

Zum einen wird in (79) ignoriert, dass in den jüdischen Gemeinden inner- und außerhalb Israels stets und ständig über israelische Politik debattiert wird und kontroverse Auseinandersetzungen geführt werden, jüdische Israel-Kritik also keineswegs eine Ausnahme ist. Zum anderen implizieren diese Modalpartikeln den rhetorischen Topos: 'Die Kritisierten sagen ja selbst ...'. Sehr häufig wird, um 
dieses Argument implizit einzuführen, explizit auf das Jüdisch-Sein der genannten Autoritäten verwiesen wie in (80).

„Vielleicht sollten Sie einmal lesen, was Uri Avneri über den Nahost-Konflikt sagt und Frau Patricia Langer, die nach ihrer Darstellung mit Mord bedroht wurde und die richtig sagt: 'Die einzige Lehre aus Auschwitz ist Gerechtigkeit.’ (Beides Juden).“[ZJD_30.03.2002_Kos_001]

Im Vergleich zu nicht-jüdischen wird jüdischen Autoritäten also eine höhere Relevanz bzw. argumentative Schlagkraft oder auch thematische Nähe oder Involviertheit unterstellt, denn wenn die „Betroffenen“ der gleichen Meinung sind, kann die eigene Einschätzung nicht falsch sein. Ganz nach dem Grundsatz Juden sagen ja selbst ..., also muss es richtig sein wird implizit konstatiert, dass Israel-Kritiker à la Avnery als positive Ausnahme die Regel bestätigen.

Gern verweisen die Schreiber auch, genau dieses Argument benutzend, auf jüdische Freunde und Bekannte, welche die gleiche (israel-kritische) Position wie sie selbst vertreten, natürlich ganz objektiv und glaubwürdig, denn sie sind ja Juden. Das Jüdisch-Sein selbst hat hier also für die Schreiber schon autoritätssteigernden Charakter und wird als eine (wie auch immer geartete) Sachkenntnis oder Befugnis gesehen. Zugleich wird dabei eine Entlastungsstrategie und implizite Antisemitismus-Abwehr realisiert, indem auf den jüdischen Bekanntenkreis verwiesen wird, um einen möglichen Antisemitismusverdacht zu verhindern. Im Unterschied zu der Schuld- oder Verantwortungsabwehr durch die Nennung des Geburtsjahrgangs („Gnade der späten Geburt“) wird der Bezug auf jüdische Freunde nicht am Anfang der Zuschrift, sondern wie in (81), der E-Mail eines promovierten Akademikers, eher am Ende des Textes realisiert.

„Wenn Sie diese Äußerung zu anmaßend finden, darf ich Ihnen noch zur Kenntnis geben, daß ich seit Jahrzehnten mehrere jüdische Familien zu meinen Freunden zählen darf, auch im Ausland.“

[ZJD_30.06.2002_Pol_001]

Neben den jüdischen Autoritäten stützen sich die Schreiber auch auf eine Vielzahl nicht-jüdischer Autoritäten, deren Äußerungen sie aus dem Kontext nehmen und je nach Funktion beliebig in ihre Argumentation einfügen.

(82) „ich habe Verständnis dafür, wie schwer es für Juden und Israelis sein muss, sich von Deutschen Kritik anhören zu müssen, das Folgende aber stammt aus der Feder eines unserer größten Dichter, Theodor Fontane, der hugenottischer Abkunft war und dem man nicht unterstellen kann, 


\begin{abstract}
Antisemit gewesen zu sein; Fontane ${ }^{26}$ schrieb dies gegen Ende des neunzehnten Jahrhunderts - in echter Fürsorge, wie ich finde: 'Ich bin von Kindesbeinen an ein Judenfreund gewesen und habe persönlich nur Gutes von Juden erfahren. Dennoch hab' ich so sehr das Gefühl ihrer Schuld, ihres grenzenlosen Übermuts, daß ich ihnen eine ernste Niederlage nicht bloß gönne, sondern wünsche. Und das steht mir fest, wenn sie sie jetzt nicht erleiden und sich jetzt nicht ändern, so bricht in Zeiten, die wir beide' - die Adressatin des Briefes ist die Äbtessin Mathilde v. Rohr - 'freilich nicht mehr erleben werden, eine schwere Heimsuchung über sie herein.’“ [ZJD_Gaza2009_17/816_Mie_001]
\end{abstract}

Diese Autoritäten haben dann in der Regel einen höheren Bekanntheitsgrad und können als „generelle moralische“ Autoritäten bezeichnet werden. $\mathrm{Zu}$ den universellen Respektspersonen zählen u. a. Mahatma Gandhi, Dietrich Bonhoeffer oder Goethe und Machiavelli. Es ist sicher kein Zufall, dass die „universellen Respektspersonen“ allesamt bereits verstorben sind.

\begin{abstract}
„Wir zitierten ihm [einem 'alten israelischen Freund' und 'HolocaustÜberlebenden', d. Verf.] eine 'neue Weisheit', die des deutschen Pfarrers Dietrich Bonhoeffer, Nazigegner und Naziopfer: 'Es gibt keinen Frieden auf dem Weg der Sicherheit. Friede muss gewagt werde.'“ [IBD_25.05.2006_Ste_001]
\end{abstract}

Eine besondere Stellung nehmen außerdem Bibelzitate, wie in (84), ein, die auf eine nicht-personelle moralische oder religiöse Autorität Bezug nehmen.

$$
\text { „In der Bibel steht 'Wer Haß säht, wird Haß ernten'.“ }
$$

[IBD_23.04.2006_Ren_001]

Der Verfasser der E-Mail in (85) kombiniert verschiedene Subformen solcher Autoritätenbeweise, um seine Kritik am Zentralrat (dessen Verhalten er als „skandalös, erschreckend, perfide“ und „unanständig“ klassifiziert) zu bekräftigen; und er präsentiert sich zudem als philosemitisch durch den Abschiedsgruß „Shalom“:

26 Es ist belegt, dass Fontane die im 19. Jahrhundert weit verbreiteten judenfeindlichen Vorurteile hegte und sie z. B. in Briefen artikulierte (s. hierzu Kap. 4.2). Interessant an dem Fontane-Zitat in (82) ist, dass dort bereits das klassische Argumentationsmuster Ich habe nichts gegen Juden, aber sie sind so verderbt realisiert ist. Der moderne Verfasser von (82) trägt dieses Muster in die Gegenwart, ohne sich dessen Brisanz bewusst zu sein. Fontane wird dafür gar zu einem wahrhaft fürsorglichen Menschen aufgewertet. 


\begin{abstract}
„Perfide vor allem deshalb, weil Sie genau wissen, dass (neben Hunderten durch die USA verhinderten Resolutionen) 9 Resolutionen bestehen, die Israel e i n d e u t i g auffordern, sich zum Beispeil auf die Grenzen des 6Tagekriegs zurückzuziehen (um nur mal eine der Resoltutionen zu nennen). Ich empfehle Ihnen statt-dessen mal die Seiten www. jews for a just peace. com und jüdische Stimme. Shalom PS: Auge um Auge, Zahn um Zahn in Ihrer Thora ist letztlich ein Gebot zur Verhältnismäßigkeit. Es heißt schließlich nicht 10 Augen für ein Auge, so wie das gegenwärtige Opferverhältnis aussieht!!“ [ZJD_24.07.2006_Sch_001]
\end{abstract}

Es lassen sich allgemein anerkannte Autoritäten von eher gruppenspezifischen Autoritäten unterscheiden. So ist Uri Avnery innerhalb links orientierter Kreise und bei Personen, die sich mit dem Themenzusammenhang Nahostkonflikt und Israel-Kritik beschäftigen, in der Regel bekannt. Für die große Mehrheit dürfte er jedoch relativ unbekannt und daher keine ausgewiesene Autorität sein.

Des Weiteren referieren die Schreiber auf politische, wissenschaftliche oder kulturelle Autoritäten wie Politiker, Professoren oder Schriftsteller, die sich israel-kritisch äußern:

„In ähnlicher Weise wie Mr. Annan haben sich jüngst auch die Ehefrau des britischen Premierministers Blair und der britische Außenminister Straw geäußert. Ich frage mich, ob nach dem Verdikt des Zentralrats der Juden nunmehr auch Mr. Anan, Mrs. Blair und Mr. Straw als Antisemiten anzusehen sind?“ [ZJD_23.06.2002_Gra_001]

„2 renommierte Professoren der Havard+ Chicago-Universitäten, Walt und Mearsheimer beschreiben unter dem Titel: The Israel-Lobby und US Foreign Policy, wie gerade in 'Nahost' von Israel die Politik vorgeschrieben wird, die die USA zu befolgen haben [...].“[IBD_10.05.2006_Hue_001]

Solche „tagesaktuellen“ Verweise auf Israel-Kritiker bilden sehr häufig den eigentlichen Anlass zum Schreiben, d. h. erst durch die Äußerungen der (jüdischen) Autoritäten, die ihnen das Argument also mehr oder weniger schon anbieten, sind die Absender motiviert, an den ZJD oder die IBD zu schreiben. Die Schreiber nehmen Kenntnis von diesen Äußerungen und haben damit einen Anlass, ihr Zustimmungsbedürfnis zu kommunizieren. Einige der Vielfach-Schreiber senden sogar immer dann einen Brief oder eine E-Mail an den ZJD oder die IBD, wenn sie einen neuen Text oder ein neues Interview von Uri Avnery oder anderen finden. Postwendend nach Erscheinen wird der entsprechende Text als Attachment mit der entsprechenden expliziten oder impliziten Zustimmung versendet. Daran 
schließt sich die Aufforderung an den ZJD oder die IBD an, diese Positionen endlich zur Kenntnis zu nehmen und danach zu handeln, wie in (88):

$$
\begin{aligned}
& \text { „Ich kann natürlich verstehen, daß die Sympathien des Zentralrats der } \\
& \text { Juden bei Israel liegen. Aber würde ihm nicht trotzdem etwas kritische } \\
& \text { Distanz zu israelischen Gewaltexzessen gut anstehen? Sie wissen sicher, } \\
& \text { wer Reuven Moskowitz ist. Wenn doch Israel sich die Weisheit zu eigen } \\
& \text { machte, die er zu seiner Lebensleitlinie erklärt hat: Ein Held ist, wer } \\
& \text { seinen Feind zum Freund macht.“ [ZJD_15.08.2006_Heu_001] }
\end{aligned}
$$

Dass die Autoritätenbeweise häufig „gerade recht kommen“ oder „verfügbar“ waren und nicht gesucht wurden bzw. eine tiefergehende Auseinandersetzung mit den Meinungen der „Autoritäten“ nicht stattfindet, wird daran ersichtlich, dass deren Namen zum Teil falsch zitiert werden (z. B. Patricia Langer statt Felicia Langer) oder Textpassagen falsch oder verkürzt wiedergegeben werden. Des Weiteren ist es natürlich immer eine Ermessensfrage, inwiefern es sich bei den einzelnen Personen, die zitiert oder genannt werden, tatsächlich um Autoritäten handelt. Häufig handelt es sich auch um Pseudoautoritäten, weil die Personen gar keine Sachkenntnis oder moralische Integrität vorweisen (so z. B. in (86), wo u. a. Cherie Blair aufgeführt wird, deren Autorität oder politisches Gewicht wohl darin bestehen soll, mit Tony Blair verheiratet zu sein).

In den meisten Fällen werden diese Verweise explizit verbalisiert, d.h. die Zitate werden mittels eines übergeordneten Matrixsatzes mit diversen sprechaktanzeigenden Verben wie sagen/beschreiben/der Meinung sein, dass ... usw. eingeleitet. Implizite Autoritätenbeweise kommen sehr selten vor, und wenn, dann auf textueller Makroebene, wenn z. B. Textpassagen einleitend oder abschließend zitiert oder ganze Artikel angehängt werden, dies aber in der Zuschrift selbst nicht kommentiert wird (hier sind vor allem vorangestellte Bibelverse oder Aphorismen zu nennen). Dabei ergibt sich aber über das für die Kommunikation basale Relevanzprinzip und die entsprechenden Implikaturen, dass die kommunikative Absicht des Verweises darin besteht, die eigene Meinung fundieren zu wollen. Das Zitieren oder Anhängen von Texten ist nur über diese Annahme plausibel zu erklären.

Auffällig ist, dass der Schluss vom als Argument angeführten Autoritätenbeweis zur eigenen Meinung fast nie expliziert wird, Formulierungen wie Ich sehe dies ebenso .../Das ist auch meine Meinung .../Somit stimmt meine Auffassung, dass ... usw. kommen kaum vor. Vielmehr wird der argumentative Schluss bzw. die Schlussregel 'meine Meinung ist fundiert und richtig, weil die Autorität/en der gleichen Meinung ist/sind' von vornherein unterstellt. Dies hängt sicherlich damit zusammen, dass der Autoritätenbeweis als eines der häufigsten rhetori- 
schen Mittel konventionalisiert ist und seine argumentative Beweiskraft oder sein Aufbau automatisch mitgedacht wird.

Bei der Bezugnahme auf Autoritäten, und in besonderem Maße bei dem Verweis auf jüdische Autoritäten, zeichnet sich insgesamt eine klare Bewertungsdichotomie ab: Juden, die Israel und seine Politik kritisieren oder boykottieren, werden als moralische Autoritäten wahrgenommen und gelobt. Die Juden, die sich mit Israel solidarisieren, den Staat verteidigen oder Kritik zurückweisen, werden angegriffen und belehrt. So erheben die vermeintlich ethisch Integren das Verhältnis zu Israel und seiner Politik bzw. die Israel-Kritik zur moralischen Gretchenfrage, der sich jeder Jude zu stellen habe.

\subsection{Relativierungsstrategien: „,Wir schreiben das Jahr 2007“}

Relativierungsstrategien basieren auf argumentativen Mustern, die der Revision und/oder Relativierung der deutschen Schuld und/oder Verantwortung auf gesamtgesellschaftlicher Ebene dienen. Bei den Relativierungsstrategien steht nicht primär die persönliche Absicherung der Äußerungen und Einstellungen des Textproduzenten (wie bei den Rechtfertigungsstrategien) im Vordergrund, sondern die allgemeine Rekonzeptualisierung der deutschen Vergangenheit. Natürlich geht aber implizit mit einer globalen Schuld- und Verantwortungsabwehr auch eine persönliche Entlastung einher. Relativierungsstrategien, von denen die Täter-Opfer-Umkehr die frequenteste ist, haben daher immer eine individuelle und eine kollektive Lesart (vgl. hierzu auch Wodak et al. 1990: 352, Bergmann/Erb 1991, Holz 2001, 2005).

Die Täter-Opfer-Umkehr-Strategie basiert auf der Konzeptualisierung DIE JUDEN SIND NICHT OPFER, SONDERN TÄTER.

Max Horkheimer hat die psychologische Dimension, die der Täter-OpferUmkehrung inhärent ist, treffend beschrieben:

„Verletzter Stolz bedeutet eine Wunde im Kollektiv nicht weniger als im Individuum. Die Juden, die die Opfer waren, sie sind mit dem Gedanken an die Katastrophe verknüpft, mit der von Deutschen wie mit der an Deutschen geübten Gewalt. Im Unbewussten werden die Rollen vertauscht. 'Nicht der Mörder, der Ermordete ist schuldig.' Narzißtische Kränkung zu überwinden, ist überaus schwer, und noch die Generation, die gar nicht beteiligt war, leidet an der Wunde, die sie selbst nicht kennt.“ (Horkheimer [1961] 51997: 314)

Allerdings muss in Ergänzung dazu festgehalten werden, dass es sich nicht immer um unbewusste Phänomene handelt. Als kommunikative Strategie wird die Täter-Opfer-Umkehr jedoch gezielt und bewusst eingesetzt. Es gibt diese Strategie in mehreren Manifestationsvarianten. Bei rechtsradikalen Schreibern und 
Neonazis realisiert sie sich entweder vergangenheitsbezogen als Verweis auf die Gefahr, die seinerzeit von den Juden für Deutschland ausging und die Notwendigkeit, sich gegen den jüdischen Feind zu wehren. Rückwirkend wird das Verhältnis von Tätern und Opfern umgekehrt.

„HITLER WAR VERRUECKT, ABER ER HAT ERKANNT DAS IHR EIN MOERDERPACK SEID. Scheiss JUDEN. IHR DRECKS-VOLK [...]“ [IBD_07.05.2010_ano_040]

(90) „HITLER hatte RECHT, Ihr seid eine kranke Rasse.“ [IBD_01.06.2010_ano_017]

(91) „HitlereHAT VOR EUCH DRECKSPACK GEWANRT.“ [IBD_01.06.2010_ano_024]

(92) „Warum wurden sie [‘die selbst ernannten Juden’, d. Verf.] denn auf der ganzen Welt verfolgt? [...] Einen Grund hatte der Herr Hitler vielleicht doch?“ [ZJD_25.07.2006_ano_007]

Ähnliche Begründungszusammenhänge werden auch von Schreibern aus der Mitte konstruiert und angeführt (s. hierzu auch Kap. 6, Bsp. (23)):

(93) „In den letzten Wochen habe ich Hitler begriffen warum er die Juden ausrotten wollte!“ [ZJD_01.09.2006_Sch_002]

(94) „Wenn alle Juden so sind, wie Sie, dann wissen wir jetzt warum die Vergangenheit in Deutschland so passiert ist.“ [IBD_19.04.2008_Bec_001]

Einer solchen Referenzialisierung folgt zumeist der Hinweis auf die aktuelle Bedrohung durch jüdische Verschwörungen sowie durch die Weltgefahr Israels.

(95) „Warum müsst ihr mit aller Macht die ganze Welt beherrschen?“ [ZJD_28.09.2007_Sch_001]

(96) „Israel kontrolliert Amerika und sicherlich morgen die ganze Welt.“ [ZJD_Gaza2009_522/816_ano_001]

(97) „Die von Euch Juden hgeheim gebaute Atombombe hast das Gleichgewicht gestört. Und Euch und den Neo-Cons ist es am ehesten zuzutrauen dass sie diese Waffe auch einsetzen.“[IBD_31.10.2006_ano_001] 
In Verbindung mit Schuld- und Verantwortungsabwehr wird diese Strategie aber auch ausschließlich gegenwartsbezogen benutzt wie in (98), der E-Mail einer 37-jährigen Lektorin und Journalistin:

\begin{abstract}
„Das deutsche Volk hat über Generationen hinweg genug Buße tun müssen - in Form von erdrückenden Steuergeldern für die Juden. Was gibt's da also zu beschweren? Euch geht's doch bestens - auf Kosten des Staates, auf Kosten von mir und meinem Mann z. B. als Steuerzahler! Ist das denn immer noch nicht genug?“ [ZJD_04.05.2005_Kar_001]
\end{abstract}

Hier dient die Täter-Opfer-Umkehr der gegenwärtigen Entlastung und entspricht dem Bedürfnis nach Normalität. Die in Deutschland lebenden Juden werden als erpresserische Holocaust-Ausbeuter in der Täterrolle dargestellt, die den leidenden Deutschen finanziell und moralisch Schaden zufügen. Auch bei dieser Variante spielt aber natürlich das Bewusstsein über den Opferstatus der Juden in der NS-Zeit eine Rolle. Dieser wird jedoch oft expressis verbis aufgehoben:

„Dieses ‘ach die armen Juden' habt ihr mittlerweile verspielt!“ [IBD_30.06.2006_Wor_001]

(100) „Treten Sie endlich aus der ewigen Opferrolle heraus und stellen sich der eigenen Verbrechen.“[ZJD_31.07.2006_Wol_001]

Krude Vermischungen von verschiedenen Referenz- und Zeitebenen wie in (101) zeigen die konzeptuelle Verschmelzung von jüdischen und israelischen Belangen:

(101) „Israel bezeichnet sich gerne als Opfer. Diese Rolle haben sie nach dem 2ten Weltkrieg beibehalten, obwohl sie mitlerweile Täter sind.“ [ZJD_13.04.2002_ano_002]

Die (gebildeten) Schreiber aus der Mitte projizieren bei der Täter-Opfer-Umkehr ihre anti-jüdischen Ressentiments weniger auf die Vergangenheit, sondern mehr auf die aktuelle Lage in Israel. Dabei spielt die Konzeptualisierung ISRAEL ALS KOLLEKTIVER JUDE eine entscheidende Rolle:

(102) „die Nachkommen der Opfer werden selbst $\mathrm{zu}$ unbeschreiblichen Tätern.“[ZJD_01.08.2006_Bah_001] 
Den in Deutschland lebenden Juden und dem Zentralrat wird Mittäterschaft an den „kriminellen Taten der Israelis“ vorgeworfen. Viele Verfasser wollen den gesellschaftlichen Druck der historischen Belastung, die als Zwang empfundene moralische Entrüstung nicht akzeptieren. Sie relativieren „ihren deutschen Makel“ durch die Konstruktion von Täterprofilen auf jüdischer Seite. Diese Täterschaft wird zugleich nach doppeltem Standard bzw. unikaler Fokussierung bewertet (vgl. hierzu auch Kap. 7.2.3). Dies bedeutet, dass die den modernen Juden unterstellten Verbrechen als besonders niederträchtig angesehen werden (da diese aufgrund ihrer Vergangenheit geläutert sein müssten):

(103) „Ausgerechnet die Juden, die so viel Leid erlitten haben, sind jetzt brutale Kriegsverbrecher.“[ZJD_28.05.2007_Blu_001]

(104) „Nach ihren eigenen schlimmen Erfahrungen eigentlich unfassbar.“ [IBD_30.11.2007_Rat_001]

Das in solchen Äußerungen enthaltene Argument, dass 'Juden heute das mit den Palästinensern tun, was die Deutschen in der Vergangenheit mit den Juden getan haben', nimmt dem Holocaust zugleich seine Unikalität und minimiert seine zivilisationsbrechende Dimension sowie das Ausmaß seiner Unmenschlichkeit. Verfasser aus der Mitte versuchen diese Semantik stets mittels Vermeidungs- und Abschwächungsstrategien zu kaschieren.

(105) „Mit Erschrecken stelle ich fest, wie in meinen eigenen Bekanntenkreis immer mehr Menschen, die man wirklich nicht als rechtsradikal einstufen kann, massive Kritik an Israel üben. Noch geschieht dies hinter vorgehaltener Hand. Aber ich fürchte, das Tabu gegenüber öffentlicher Kritik an Israel könnte leicht zerbrechen. Die Sympathie einer ganzen Generation für Israel und für das jüdische Volk ist dabei, sich in Entsetzen und Enttäuschung aufzulösen. Es wird immer schwerer zu verstehen, wie ein Volk, das selbst eine lange Leidensgeschichte durchlebt hat, einem anderen Volk so viel Leid zufügen kann.“ [ZJD_01.05.2002_Sch_001]

Solche Schreiben kodieren den unzulässigen konzeptuellen Transfer ISRAELISCHE TATEN SIND JÜDISCHE TATEN, sie relativieren damit zugleich vergangenheitsbezogen die deutschen Verbrechen, sie konstruieren de-realisierend moralische Verkommenheit auf jüdischer Seite, und sie rechtfertigen ihre eigene, aber anderen in den Mund gelegte Abneigung gegenüber Juden, da diese als schlimme Täter gesehen werden. 
Gekoppelt an die Täter-Opfer-Umkehr finden sich mehrere Argumentationsmuster, die in Relativierungsstrategien eingebunden sind: Argumentativ erfolgt die Revision der deutschen Schuld bei den untersuchten Zuschriften immer über die Form des Geschichtsrevisionismus, s. a). Hingegen werden bei einer Relativierung der deutschen Schuld und/oder Verantwortung, s. b), sowie bei einer Relativierung deutscher Verantwortung unter Anerkennung der deutschen Schuld, s. c), die historischen Fakten der NS-Geschichte nicht geleugnet, sondern in ihrer heutigen Relevanz infrage gestellt. Eine Relativierung und Abwehr der deutschen Verantwortung resultiert des Weiteren auch aus der Leugnung des aktuell in Deutschland auftretenden Antisemitismus bzw. aus dessen Marginalisierung als Randerscheinung, s. d).

a) Die Revision deutscher Schuld erfolgt in (106) explizit auf der Wortebene durch die Leugnung der NS-Verbrechen mittels des Determinativkompositums Auschwitz-Lüge auf der Basis von Lüge mit dem Bestimmungswort Auschwitz. Als einer der schlimmsten Orte der NS-Verbrechen an Juden steht Auschwitz synonym für die Gesamtverbrechen der Nationalsozialisten an der jüdischen Bevölkerung (vgl. Eitz/Stötzel 2007: 25, Chiffre Auschwitz). Solche manifesten Holocaustleugnungen und Rekonzeptualisierungen der deutschen Vergangenheit finden sich vor allem bei rechtsradikalen Schreibern, die meist anonym bleiben:

„Auschwitz-Lüge“[IBD_2004_ano_Postkarte]

„Ach, ja, ganz vergessen, die Juden wurden ja vergast, und diese 'Tatsache’ müssen wir alle glauben, dafür sorgen die BRD-Gerichte.“ [IBD_16.01.2009_ano_008]

Neben der globalen Revision treten auch partikulare Relativierungen und FaktenZurückweisungen wie in (108) auf:

(108) „Aus der Haut ermordeter Gefangener fertigte man zynischerweise Gebrauchsgegenstände, wie Lampenschirme für SS-Angehörige [...]' Diese Geschichte ist ein Lügenmärchen! [...] das Lampenschirmmärchen.“[ZJD_25.04.2007_Sch_001]

Die Tatsache, dass aus der Haut von ermordeten Gefangenen Lampenschirme für SS-Angehörige gefertigt wurden, wird explizit über das Determinativkompositum Lügenmärchen als falsch dargestellt.

Auffällig ist die Spezifizierung der Kompositionsbasis Märchen über das Bestimmungswort Lüge, denn hier werden zwei semantisch nahe stehende Kon- 
zepte - die semantische Gemeinsamkeit besteht in der fiktiven Komponente miteinander verknüpft. Im Unterschied zum Märchen, bei dem die Fiktionalität erwartet und akzeptiert und somit positiv bewertet ist, ist mit dem Konzept LÜGE eine Negativbewertung und Sanktionierung der geäußerten Unwahrheit verbunden.

b) Eine Relativierung deutscher Schuld kann auf der Satzebene wie in Beispiel (109) über die Aufrechnung von eigenem Leid mit dem Leid der Opfer des Holocaust erfolgen.

(109) „Ich bin keine Antisemitin und auch unsere Familie hat unter dem 3. Reich gelitten!“ [ZJD_06.04.2002_Ses_001]

Dieser Aussage liegt die kausal konstruierte Beziehung zugrunde: 'Weil meine Familie ebenfalls Leid erfahren hat, kann ich keine Antisemitin sein' und das impliziert wiederum 'wenn man selbst einer Opfergruppe angehört(e), kann man heute kein Täter sein.’

Im Beispiel (110) erfolgt eine implizite Relativierung deutscher Verantwortung und Schuld auf Textebene über die Verwendung rhetorischer Fragen und einer Analogiekonstruktion sowie über den Gebrauch von NS-Vergleichen. ${ }^{27}$ Die Äußerung ist auf der Wortebene darüber hinaus durch Übertreibung und Verharmlosung sowie Vagheit gekennzeichnet:

(110) „Warum vergießt Ihr alle Krokodilstränen, wenn mal jemand einen Gegenstand auf eine jüdische Synagoge wirft, gleichzeitig aber schweigt Ihr, wenn unsere Geburtskirche in Bethlehem, von den jüdischen Militärhorden zerstört wird ? Was müssen die jüdischen Nazihorden denn noch unternehmen, um deutsche 'Persönlichkeiten' zu einer Stellungnahme zu bewegen?“'[ZJD_08.04.2002_Sch_001]

Mittels des Kompositums Krokodilstränen wird die öffentliche (deutsche) Reaktion auf die anti-jüdische Anschlagsserie in Frankreich und Belgien in der Osterzeit $2002^{28}$ als heuchlerisch charakterisiert. Das Ausmaß der Anschläge wird verharmlosend dargestellt, indem vage mittels der Modalpartikel mal, dem Indefinitpronomen jemand und der unterspezifiziert bleibenden indefiniten Nomi-

27 Zu den Spezifika des NS-Vergleichs s. Kap. 7.2.2.

28 Es wurden vielerorts Synagogen, Geschäfte und Schulen in Brand gesetzt, Friedhöfe geschändet, Synagogen und Menschen mit Steinen beworfen (vgl. NZZ am 02.04.2002, S. 3 oder Tagesspiegel am 04.04.2002, S. 7). 
nalphrase einen Gegenstand auf das Ereignis referiert wird. Zugleich erfolgt eine falsche Sachverhaltsrepräsentation durch die Behauptung, es sei nur eine Synagoge mit einem Gegenstand beworfen worden. Eine derart verzerrende Perspektive marginalisiert die antisemitischen Ereignisse. Kontrastiv wird der israelische Militäreinsatz, der der Befreiung der von bewaffneten Palästinensern belagerten Geburtskirche in Bethlehem diente, als Zerstörung bewertet; die Einsatzkräfte werden als Militärhorden und Nazihorden beschimpft. Mit dieser Israel als Täter dämonisierenden Perspektive geht wiederum eine de-realisierende Sachverhaltsrepräsentation einher, denn es wird behauptet, dass die Geburtskirche vernichtet worden sei (das Verb zerstören impliziert ein großes Ausmaß an Vernichtung). Des Weiteren ist ein Bruch bezüglich der Informationsstruktur zu erkennen, denn im Gegensatz zu den antijüdischen europäischen Ereignissen werden bei den israelischen die vermeintlichen israelischen „Täter“ benannt und ausschließlich ihr Verhalten fokussiert. Auffällig ist die Lexemwahl unsere Geburtskirche. Mit Hilfe des Possessivpronomens wird eine religiös begründete Wir-Gruppe der Christen konstruiert, von der die Israelis als Ihr-Gruppe ausgeschlossen sind und der die Konzeptualisierung JUDEN = ISRAELIS zugrunde liegt.

Monoperspektivische Darstellungen von Ereignissen, wie in diesem Beispiel, die sich durch Verkürzung komplexer Sachverhalte und Aufgreifen einzelner brisanter und emotionaler Details kennzeichnen, die wiederum in eine in sich schlüssige (wenn auch realitätsferne) Argumentation passen, sind typischer Ausdruck eines konzeptuell geschlossenen Textweltmodells (vgl. Schwarz-Friesel 2007: 226).

c) Eine Relativierung deutscher Verantwortung kann auch unter Anerkennung der deutschen Schuld stattfinden, wie beispielsweise in (111) und (112) über eine explizite Schlussstrichforderung.

„Wir schreiben das Jahr 2007 und nicht mehr 1945. Letzteres ist fast zwei Generationen her. Was damals geschehen war, soll in keiner Weise relativiert werden. Aber: Es reicht!“ [ZJD_16.04.2007_Sch_003]

Argumentativ wird die Aufforderung, Ermahnung und Erinnerung zu beenden, lediglich mit dem langen Zeitabstand zu den NS-Verbrechen begründet.

In (112) wird die Schlussstrichforderung vage mittels der Gradpartikel genug begründet, und dieser Forderung wird durch das Modalverb müssen und die Modalpartikeln endlich und mal Nachdruck verliehen.

(112) „Deutschland hat genug für Israel und die Juden getan und damit muß entlich mal schluss sein .“[ZJD_13.05.2007_Lud_001] 
d) Während in a) bis c) die Relativierung der deutschen Vergangenheit im Vordergrund steht, um die Verantwortung der Deutschen abzuwehren, zielt ein weiteres argumentatives Muster darauf, das Vorhandensein von Antisemitismus in der Gegenwart zu leugnen, wie in (113), bzw. zu relativieren, wie in (114). Solche Äußerungen fokussieren weniger die individuelle Einstellung als vielmehr eine angebliche gesamtgesellschaftliche Vorurteilsfreiheit. Der Textproduzent versteht sich aber immer auch als Teil der Gesellschaft und grenzt sich somit von einem Antisemitismus-Vorwurf ab. Das Ausmaß des Antisemitismus wird hierbei marginalisiert und die gesellschaftliche Relevanz der Auseinandersetzung mit Antisemitismus verneint (s. hierzu auch die Beispiele (182) ff. in Kap. 5.2).

$$
\text { „Antisemitismus ist hier unbekannt.“ [IBD_23.07.2006_Her_002] }
$$

So wird beispielsweise in (113) der aktuelle Antisemitismus explizit geleugnet oder wie in (114) als rechtsextreme Randerscheinung marginalisiert sowie im Verhältnis zu Israels „Untaten“ als harmlos charakterisiert, s. (115). Häufig wird auch eine rechtsextreme Einstellung als solche als ungefährlich erachtet und in diesem Zusammenhang relativierend auf einen israelischen Rassismus verwiesen.

(114) „Diese Aktivitäten in Israel scheinen mir derzeit um Einiges brisanter fuer den Weltfrieden zu sein als neonazistische Umtriebe in Deutschland.“ [ZJD_24.10.2006_Bud_001]

(115) „Von wegen rechtsradikale Vorboten eines neuen Hitler; QUATSCH. Viel harmloser sind diese Leute.“ [ZJD_24.10.2007_ano_003]

Bei diesen Formen des Entlastungsantisemitismus lassen sich zudem drei unterschiedliche Konzeptualisierungen unterscheiden, die Juden die Schuld geben (s. auch Kap. 5): a) JUDEN FÖRDERN GENERELL DURCH IHR VERHALTEN ANTISEMITISMUS, vgl. (116), b) JUDEN IN DEUTSCHLAND FÖRDERN ANTISEMITISMUS DURCH IHRE POSITIONIERUNG GEGENÜBER ISRAEL, vgl. (117), und c) ISRAEL(ISCHE POLITIK) IST SCHULD AM ANTISEMITISMUS IN DEUTSCHLAND, vgl. (118). So befindet ein Beamter, der seit 30 Jahren bei der Bundeswehr arbeitet:

(116) „irgendwie entsteht in der brd ein neuer judenhass - auch dank ihrer mithilfe.“ [ZJD_02.05.2007_Moh_001]

Ein Bürger aus Hildesheim verknüpft seine Beschwerde mit einem entsprechenden Vorwurf, der einen direkten kausalen Zusammenhang konstatiert: 
„Es ist einfach niederträchtig von Ihnen, jedes Wort der Kritik an israelischer Politik mit Antisemitismus gleichzusetzen. Sind Sie sich eigentlich bewußt darüber, dass Sie mit Ihren Äußerungen erst das hervorrufen, was Sie beklagen?““[ZJD_02.09.2006_Pap_001]

Ein Bedburger gibt die Erklärung expressis verbis formuliert: Antisemitische Aktivitäten resultieren einzig aus dem intolerablen Verhalten aller Israelis.

(118) „Und sie beschweren sich über die wachsende Judenfeindliche Handlungen in Europa? Das sind sie selber schuld. [...] Denn die Israelis betreiben Rassismus, Fremdenfeindlichkeit und Intoleranz“

[IBD_04.07.2007_Dro_001]

Gleichzeitig werden in diesem Zusammenhang genuine antisemitische Tendenzen der Deutschen geleugnet oder relativiert, und damit wird die Verantwortung abgelehnt bzw. verschoben.

(119) „Wissen Sie, Herr Kramer, dass der so oft zitierte Antisemitismus im Grunde überhaupt kein Gewicht hat in der Bevölkerung? Wenn der Zentralrat der Juden behauptet, der Antisemitismus in Deutschland wachse und bezieht sich dabei auf 200 lächerliche Mails diverser Online-Junkies, halte ich das für wenig verantwortungsbewusst.“

[ZJD_27.07.2006_Gro_001]

(120) „Aber vielleicht brauchen Sie das ja, um Ihr Weltbild zu bestätigen, dass Deutschen “antisemitisch” eingestellt seien.“[ZJD_30.07.2006_Cla_001]

Die Bagatellisierung des aktuellen Antisemitismus wird dabei stets durch die Behauptung vorgenommen, dass es keine (oder keine ernstzunehmende) Judenfeindschaft in der deutschen Mehrheitsgesellschaft gebe.

\subsection{Abgrenzungsstrategien: „Sie sind eine Truppe“}

Die Ab- und Ausgrenzung von Juden als Ihr-Gruppe (Out-Group) ist eine semantische Konstante im antisemitischen Diskurs, die über Kontrastierungen realisiert wird (vgl. hierzu bereits Kap.5). Dies geschieht zum einen innerhalb der bereits genannten Textstrategien und zum anderen über spezifische Abgrenzungsmuster. Auf der sprachlichen Ebene zeichnen sich die jeweiligen argumentativen Muster durch eine Oppositionssetzung von Deutschen und Juden bzw. Israelis 
aus. Auffälliges Charakteristikum ist die Konzeptualisierung JUDEN = ISRAELIS und die damit einhergehende Synonymverwendung der Lexeme Jude(n) und Israeli(s), die oftmals mit einer monoperspektivischen Wahrnehmung von Israel als einem Aggressorstaat einhergeht.

Da Abgrenzungsstrategien auf Formen der Kontrastierung bzw. Gegenüberstellung hinauslaufen, können sie auf vielfältige Weise sprachlich realisiert werden und im gesamten Text auftreten. Im Folgenden werden die geläufigsten Varianten aufgeführt.

Auf der Wortebene wird die Gegenüberstellung durch die Verwendung von Pronomina der 3. Person Singular oder Plural in Verbindung mit abfälligen Bezeichnungen realisiert, s. (121) bis (123):

„[...] ihre Vereinigung [...] Sie Herr Spiegel und ihre Organisation“ [ZJD_12.06.2002_Sta_001]

„Eure geldgierige Truppe.“[ZJD_04.05.2006_Kel_001]

„Ihrem widerlichen Lobbyiistenverband.“[ZJD_17.03.2002_Lue_001]

Oft wird diese Kontrastierung zusätzlich über die Verwendung von Pronomina der 1. Person Singular oder Plural für die Wir-Gruppe, zu der sich der Textproduzent zählt, ausgedrückt. Dies geschieht kontrastiv zu den Pronomina der 3. Person Singular oder Plural für die Ihr-Gruppe, in der Juden und/oder Israelis vom Textproduzenten verortet werden (zu inklusivem versus exklusivem Wir vgl. Mautner 1998).

„[...] ich frage mich, was sie diese trauerrede angeht.“

[ZJD_16.04.2007_Moh_001]

Der Textproduzent von (124), ein Beamter, bringt so sein Unverständnis über kritische Kommentare des Zentralrats zur Rede des damaligen baden-württembergischen Ministerpräsidenten Günther Oettinger anlässlich des Todes des ehemaligen NS-Marinerichters Hans Filbinger zum Ausdruck. Über die rhetorische Frage und die Verwendung der Pronomina ich versus sie wird die Diskussion über die Trauerrede implizit als Angelegenheit der (deutschen) Wir-Gruppe außerhalb des Kompetenzbereichs des Zentralrats als Vertreter der (jüdischen) Ihr-Gruppe konzeptualisiert. Dieser Konzeptualisierung liegt das Stereotyp JUDEN SIND KEINE DEUTSCHEN zugrunde. 
Häufig wird die Ausgrenzung von Juden als Nicht-Deutsche und von Israelis als Unmenschen auf der Wortebene mittels stark pejorativer Adjektive und Nomina ausgedrückt (s. hierzu Kap. 10.1):

„widerwärtiges israelisches Pack [...] Ihr Rattenpack“ [IBD_08.05.2007_Kol_001]

(126) „kranke, widerwärtige und ultrabrutale Wichser“ [IBD_10.06.2006_Bre_001] „Mitleidlose Kreaturen seid Ihr.“[ZJD_01.08.2006_Mon_001]

Dehumanisierung (z. B. durch Tierbezeichnungen) und moralische Ab- und Entwertung sind hierbei dominant.

Auf der Satzebene erfolgt die Ab- und Ausgrenzung auch explizit mittels direktiver Sprechakte wie Aufforderungen:

„halten sie sich zurück mit ihrer kritik an deutschen aktivitäten im ausland. das ist eine einmischung in innere angegelenheiten des deutschen staates, der ihnen als nicht-europäische sekte nicht zusteht.“ [ZJD_29.05.2007_Sch_002]

In Beispiel (129) lässt allein die Anhäufung rhetorischer Fragen und die Verwendung der Modalpartikel eigentlich auch ohne kontrastiv gesetzte Pronomina den Schluss zu, dass der Textproduzent in Deutschland lebende Juden nicht als Deutsche konzeptualisiert. Dass diese Schlussfolgerung gerechtfertigt ist, wird im nachfolgenden Satz deutlich.

(129) „Was sind die Mitglieder des ZENTRALRATES eigentlich: Jüdische Bürger in Deutschland ? Deutsche Juden ? Israelische Juden in Deutschland ? Letztere Deutung liegt nahe, verhalten sich doch Frau Knobloch und Herr Korn - wie ehedem die Herren Bubicz und Spiegel - gegenüber deutschen Politikern und Bürgern wie die '5. Kolonne' eines fremden Staates ?“ [ZJD_02.11.2006_Buh_001]

Zwar sind Ab- und Ausgrenzungsstrategien, die auf tradierten judeophoben Stereotypen basieren, besonders oft bei rechtsradikalen Verfassern zu beobachten, doch auch gebildete Schreiber greifen auf diese Kontrastierungsmuster zurück. So wie in (130), wo ein promovierter Akademiker aus Lauf die deutschen Juden als Israelis (Ihres Landes) sieht: 
„Statt gekränkt über Antisemitissmus zu reden, sollte Herr Friedmann Format zeigen und sich Gedanken machen, ob nicht doch ein bisl Wahrheit in der Kritik Möllemanns steckt. Vornehme Zurückhaltung, gerade in der jetzt so schwierigen Lage Ihres Landes, würde dem guten deutsch jüdischen Verhältnis dienlich sein.“ [ZJD_07.06.2002_Ban_001]

Ein immer wiederkehrendes argumentatives Muster, das der Abgrenzung von Juden dient, ist die 'moralische Diskreditierung', die bei rechtsradikalen Schreibern direkt ausgedrückt wird, während Verfasser aus der Mitte dies über die Referenz auf Israel vollziehen.

Ein Schreiber aus Kornwestheim grenzt in seiner E-Mail an den Zentralrat Israel als ein barbarisches Land von anderen Ländern ab, indem er mittels diverser Vergleiche dessen angebliche Verkommenheit fokussiert und damit seine Hoffnung auf Gewalt und Leid legitimiert.

„Israel betreibt den Holocaust an den Bewohnern Palestinas. [...] Es wird offenbar, daß Israel eben nicht zum Kreis der aufgeklärt humanistischen Völker zählt, sondern kulturtell und geistig-moralisch im barbarischen Zustand verblieben ist. Es ist $\mathrm{zu}$ hoffen, daß Israel genügend Tote $\mathrm{zu}$ beklagen hat, wie Europa im 30-jährigen Krieg, um (im wahersten Sinne des Wortes) zur Vernunft zu kommen.“

[ZJD_Gaza2009_552/816_Mad_001]

Häufig wird dabei (vor allem von linksextremen und linken Verfassern) der Nahostkonflikt allein als Resultat der israelischen Politik simplifiziert und dies einseitig (monoperspektiviert) in Form von Aussagesätzen als Fakt dargestellt:

„Gerade die Hisbollah ist doch eine Gründung infolge fortgesetzten Staatsterrorismus der israelischen Regierung und des Militärs gegen seine Nachbarstaaten. Immerhin ist es in jüngster Vergangenheit der Hisbollah zu verdanken, daß die profaschistische israelische Soldateska nicht wieder bis in das wehrlose Beirut vordringen konnte und mit Panzern eine Schneise des Todes und der Zerstörung durch die Flüchtlingslager Sabrah und Schatila wie vor einigen Jahren unter General Scharon zog.“ [ZJD_07.10.2006_Zim_001]

Kennzeichnend für die monoperspektivische Darstellung des Verfassers aus (132) sind auf der Textebene eine einseitige Informationsstruktur und auf der Wortebene Negativ-Referenzialisierungen Israels. Israel wird über Lexeme wie Staatsterrorismus, profaschistisch, Schneise des Todes, Zerstörung kriminalisiert 
und dämonisiert. Hingegen wird die Terrororganisation Hisbollah nicht mit den Konzepten TERROR, TOD, UNRECHT oder SCHULD assoziiert, sondern vielmehr als BESCHÜTZER konzeptualisiert. Es findet also eine eklatante De-Realisierung statt.

Wenn Juden und Israelis prinzipiell als moralisch verwerflich oder als außerhalb der ethischen Werte und Normen stehend dargestellt sind, lassen sich daran vielfältige antisemitische Argumente und Forderungen anschließen. So können auf dieser Basis auch stets die deutsche Schuld und Verantwortung für den Holocaust und seine Folgen relativiert und geleugnet, der israelische Staat delegitimiert sowie die judenfeindliche Einstellung gerechtfertigt werden. Die den Juden unterstellte Amoralität dient als eine variabel einsetzbare Prämisse für jedwede antisemitischen bzw. anti-israelischen Meinungsäußerungen und Argumentationsketten.

Die geläufigste Form der moralischen Diskreditierung erfolgt über NS-Vergleiche. So erfolgt z. B. in (133) mithilfe der Täter-Opfer-Umkehr-Strategie durch den impliziten NS-Vergleich eine Schuldrelativierung und/oder Verantwortungsabwehr:

„Wiso wird denn über 60 jahre nach dem Ende des Nazionalsozialismus immer noch so viel unnötiges gerede über diese lang abgehandelte Zeit gehalten? Räumt doch erst mal in eurem eigenen Land auf. Was passiert denn da mit den Palestinensern?“ [ZJD_13.04.2007_ano_001]

Solchen an den Zentralrat gesendeten Texten ist stets die konzeptuelle Gleichsetzung von ISRAELIS und JUDEN inhärent. Deutsche Juden werden als Nicht-Deutsche von der Mehrheitsgesellschaft abgegrenzt. Eine moralische Diskreditierung erfolgt dabei häufig gekoppelt an das Anlegen eines doppelten moralischen Standards an Juden, wie in der E-Mail eines Diplomingenieurs aus Oberbayern:

„Trotz ihrer leidvollen Vergangenheit scheint ihnen ein Mitgefühl für die nöte anderer Völker fremd zu sein“ [ZJD_12.12.2006_Oeh_001]

Dieser Doppelstandard basiert auf der wie folgt beschreibbaren Konzeptualisierung: 'Menschen, denen Leid zugefügt wurde, müssen geläutert sein und sensibel für das Leid anderer. Die Juden aber sind moralisch besonders verkommen und roh, denn sie haben keine Lehre aus der Vergangenheit gezogen.' Die israelische Politik wird als Verstoß gegen die Normen der Wir-Gruppe (westliche demokratische Gesellschaften) allen Juden angelastet und somit als Begründung für die Ausgrenzung von Juden herangezogen (vgl. hierzu auch Holz 2005: 23-53).

Abgrenzungsstrategien beruhen insgesamt konzeptuell und emotional maßgeblich auf tradierten judenfeindlichen Stereotypen. Juden werden dabei immer 
als das Andere, das Böse und Verkommene dargestellt. Abgrenzungsstrategien dienen den antisemitischen Schreibern dazu, ihren eigenen Antisemitismus als legitim zu präsentieren und $\mathrm{zu}$ artikulieren.

Damit schließt sich ein argumentativer Kreis (judenfeindlichen Denkens und kommunikativen Handelns) in Bezug auf die erörterten Strategien: Die Ausgrenzung von Juden muss - dem antisemitischen Denken zufolge - sein, denn sie ist für die Sprachproduzenten (pseudo-)kausal begründet, wie die scheinbar rechtfertigenden Argumente zeigen, die durch die Legitimierung der eigenen Person und Position abgesichert sind. Zugleich muss sichergestellt sein, dass der Eindruck vermieden wird, man sei ein Rassist oder Antisemit. Offensive Selbsterhöhung und defensive Selbstverteidigung interagieren bei dem Versuch, das konzeptuell geschlossene Weltbild aufrechtzuerhalten und überzeugend an andere zu vermitteln.

\section{Rechts versus Mitte}

Die verbalen Strategien treten in den untersuchten Zuschriften nicht gleichmäßig verteilt auf. Vielmehr gibt es auffällige Unterschiede zwischen extremen und eher gemäßigten Zuschriften. Legitimations- und Vermeidungsstrategien treten besonders häufig bei Textproduzenten auf, die sich selbst in der gesellschaftlichen Mitte oder dem politischen Mainstream verorten. Die exemplarischen Analysen zeigen, dass Verfasser, die eliminatorischen bzw. vulgär-rechten Antisemitismus artikulieren, auf diese Strategien überwiegend verzichten. Rechtsextreme Schreiber halten es also für unnötig, ihre antisemitischen Meinungen zu kaschieren oder argumentativ zu begründen. Zugleich finden sich bei (den wenigen) Schreibern, die tatsächlich nur legitime Kritik an Israel üben, kaum Legitimations- und Vermeidungsstrategien. Dies deutet darauf hin, dass sich Verfasser, die anti-israelische und antisemitische Positionen vertreten, durchaus im Klaren darüber sind, dass ihre Meinungen brisant und zweifelhaft bzw. zumindest diskussionswürdig und angreifbar sind. Ohne dieses Bewusstsein gäbe es keine Motivation für die Verwendung der Strategien. Daher sind das Fehlen bzw. das Vorhandensein der erörterten Strategien wichtige Indikatoren für Verbal-Antisemitismus bei uneindeutigen oder stark impliziten Äußerungen und zwar insbesondere dann, wenn sie der kommunikativen Funktion der Antisemitismus- oder Sanktionsabwehr dienen. 


\section{Fazit}

Die Analyse der Zuschriften von Schreibern aus der gesellschaftlichen Mitte zeigt (bei allen individuellen Unterschieden zwischen den Verfassern) eine erstaunlich große Homogenität in Bezug auf den textuellen Aufbau, die Verwendung spezifischer Strategien und argumentativer Muster. Die E-Mails und Briefe lesen sich mehrheitlich wie Abschriften mit geringfügigen Variationen zu einer gemeinsamen Vorlage. Inhalte und sprachliche Formen sind oft nahezu austauschbar. Es wird erkennbar, wie stark und überindividuell zum einen die judeophoben Stereotype mental präsent und einflussreich sind, zum anderen, wie der antisemitische Sprachgebrauch im 21. Jahrhundert vom Bewusstsein der Katastrophe des Holocaust geprägt ist. Den (gebildeten) Verfassern der judenfeindlichen Texte ist bewusst, dass ihre Äußerungen brisant und/oder moralisch zweifelhaft sind und/ oder als solche wahrgenommen werden könnten. Doch der Wunsch, das unbedingte Bedürfnis, sich mit ihrer empfundenen Wut und Empörung gegenüber dem ,jüdischen Störenfried“, dem „Gegner und Feind“ (in diesen Fällen verkörpert durch den Zentralrat und die Botschaft) artikulieren zu wollen, ist stärker als die Bedenken, die ihnen bei dieser Artikulation kommen. Somit zeigt sich auch bei gebildeten Antisemiten aus der Mitte der Gesellschaft die für das gesamte Phänomen des Antisemitismus charakteristische obsessive Komponente.

Der vor anderen und sich selbst geleugnete Antisemitismus erhält das Wunschbild des vorurteilsfreien, verantwortungsbewussten Bürgers aufrecht. Diese Konstellation ist (aufgrund der besonderen Geschichte) typisch für den deutschen Diskurs. Die Verfasser antisemitischer Schreiben, die der Mitte zuzuordnen sind, verschlüsseln ihre judenfeindlichen Vorurteile oft mittels impliziter oder re-klassifizierter Sprachformen und benutzen verschiedene Typen von Legitimierungs- und Rechtfertigungsstrategien, um ihre semantisch radikale Argumentation einerseits $\mathrm{zu}$ rechtfertigen und anderseits mittels verbaler Kodierung konzeptuell umzudeuten. Die Schreiber präsentieren sich selbst als Anti-Antisemiten, deren Gewissen, moralische Integrität und Verantwortungsbewusstsein es verlangen, Stellung zu beziehen gegen die aus ihrer Sicht moralisch verwerflichen Juden und/oder Israelis. Dieser „Antisemitismus ohne Antisemiten“ gibt sich anti-rassistisch und ehrbar, bedient sich aber nahezu aller gängigen judenfeindlichen Stereotype und Vorurteile, benutzt dämonisierende NS-Vergleiche und artikuliert sich über einen extremen Anti-Israelismus. Diese Kennzeichen sind auch typisch für die extremistischen E-Mail-Schreiber.

Hier liegen klar die Gemeinsamkeiten zwischen Verbal-Antisemitismus von Rechts- und Linksextremisten sowie Sprachproduzenten aus der Mitte: Zwar benutzen Extremisten mehrheitlich eine vulgäre Beschimpfungslexik sowie Bedrohungssyntax und greifen auf eine eher primitive Diffamierungsrhetorik 
zurück. Blickt man jedoch auf die tiefer liegenden Denkmuster und Ressentiments, offenbaren sich die gleichen semantisch-konzeptuellen Inhalte. Diese werden beeinflusst von basalen Stereotypen und Weltdeutungsschemata.

Trotz aller ideologischen Differenzen ist der Sprachgebrauch der meisten Verfasser erstaunlich ähnlich und weist bis in die argumentative Detailstruktur der Texte hinein grundlegende Muster auf. Nicht nur die konzeptuellen Grundlagen der judenfeindlichen Sprache, sondern auch die kommunikativen Realisierungsstrategien sind über alle politischen und sozialen Unterschiede hinweg weitgehend homogen. 